\title{
Minkowski Measurability and Exact Fractal Tube Formulas for $p$-Adic Self-Similar Strings
}

\author{
Michel L. Lapidus, Lũ' Hùng, and Machiel van Frankenhuijsen
}

\begin{abstract}
The theory of $p$-adic fractal strings and their complex dimensions was developed by the first two authors in [17, 18, 19, particularly in the self-similar case, in parallel with its archimedean (or real) counterpart developed by the first and third author in [28. Using the fractal tube formula obtained by the authors for $p$-adic fractal strings in [20, we present here an exact volume formula for the tubular neighborhood of a $p$-adic self-similar fractal string $\mathcal{L}_{p}$, expressed in terms of the underlying complex dimensions. The periodic structure of the complex dimensions allows one to obtain a very concrete form for the resulting fractal tube formula. Moreover, we derive and use a truncated version of this fractal tube formula in order to show that $\mathcal{L}_{p}$ is not Minkowski measurable and obtain an explicit expression for its average Minkowski content. The general theory is illustrated by two simple examples, the 3 -adic Cantor string and the 2-adic Fibonacci strings, which are nonarchimedean analogs (introduced in 17, 18) of the real Cantor and Fibonacci strings studied in $\mathbf{2 8}$.
\end{abstract}

\section{Contents}

1. Introduction

2. $p$-Adic Numbers

3. $p$-Adic Fractal Strings 3

4. Volume of Inner Tubes 6

5. Explicit Tube Formulas for $p$-Adic Fractal Strings 8

6. Nonarchimedean Self-Similar Strings 9

2000 Mathematics Subject Classification. Primary 11M41, 26E30, 28A12, 28A80, 32P05, 37P20; Secondary 11M06, 11K41, 30G06, 46S10, 47S10, 52A38, 81Q65.

Key words and phrases. Fractal geometry, $p$-adic analysis, $p$-adic fractal string, $p$-adic selfsimilar string, lattice string, strongly lattice string, geometric zeta function, complex dimensions, Minkowski dimension, Minkowski measurability, average Minkowski content, fractal tubes formulas, Cantor and Fibonacci strings.

The research of the first author (MLL) was partially supported by the US National Science Foundation under the grant DMS-0707524 and DMS-1107750, as well as by the Institut des Hautes Etudes Scientifiques (IHES) where the first author was a visiting professor in the Spring of 2012 while this paper was completed.

The research of the second author (LH) was partially supported by the Trustees' Scholarly Endeavor Program at Hawai'i Pacific University.

The research of the third author (MvF) was partially supported by the Georg-AugustUniversität Göttingen and the College of Science and Health of Utah Valley University. 
7. Geometric Zeta Function of $p$-Adic Self-Similar Strings 13

8. Rationality of the Geometric Zeta Function 14

9. Exact Tube Formulas for $p$-Adic Self-Similar Strings 17

10. The Average Minkowski Content 19

References

\begin{abstract}
Nature is an infinite sphere of which the center is everywhere
\end{abstract} and the circumference nowhere. Blaise Pascal

\title{
1. Introduction
}

In this paper, we present and use the explicit tube formulas obtained in [20, for general $p$-adic fractal strings, in order to derive exact fractal tube formulas for $p$-adic self-similar fractal strings. The general results are illustrated in the case of suitable nonarchimedean analogs of the Cantor and the Fibonacci strings. Some particular attention is devoted to the 3-adic (or nonarchimedean) Cantor string (introduced and studied in [17, an appropriate counterpart of the archimedean Cantor string, whose 'metric boundary' is the 3-adic Cantor set [17), a suitable $p$-adic analog of the classic ternary Cantor set. We also derive an explicit expression for the average Minkowski content of a $p$-adic self-similar string and the 'boundary' of the associated nonarchimedean self-similar set.

We note that $p$-adic (or nonarchimedean) analysis has been used in various areas of mathematics (such as functional analysis and operator theory, representation theory, number theory and arithmetic geometry), as well as (sometimes more speculatively) of mathematical and theoretical physics (such as quantum mechanics, relativity theory, quantum field theory, statistical and condensed matter physics, string theory and cosmology); see, e.g., [2, 3, 4, 11, 36, 40, 41] and the relevant references therein. In particular, ultrametric structures have been shown to be very useful tools to study spin glasses in condensed matter physics; see [36] for a comprehensive survey on this and related topics. We also point out the more recent review article 3 ] which discusses a variety of potential applications of $p$-adic analysis in mathematical physics and biology. Furthermore, several physicists and mathematical physicists have suggested that the small scale structure of spacetime may be fractal; see, e.g., [6, 8, 15, 31, 42. In addition, it has been suggested (in 41 for example) that seemingly abstract objects such as nonarchimedean fields (including the field of $p$-adic numbers) can be helpful in order to understand the geometry of spacetime at sub-Planckian scales.

Finally, we note that $p$-adic fractal strings (and their possible quantized analogs) may be helpful to obtain an appropriate adelic counterpart of ordinary (real) fractal strings, along with their quantization (called fractal membranes), as introduced in [15.

\section{2. $p$-Adic Numbers}

Given a fixed prime number $p$, any nonzero rational number $x$ can be written as $x=p^{v} \cdot a / b$, for integers $a$ and $b$ and a unique exponent $v \in \mathbb{Z}$ such that $p$ does not divide $a$ or $b$. The $p$-adic absolute value is the function $|\cdot|_{p}: \mathbb{Q} \rightarrow[0, \infty)$ given 
by $|x|_{p}=p^{-v}$ and $|0|_{p}=0$. It satisfies the strong triangle inequality: for every $x, y \in \mathbb{Q}$

$$
|x+y|_{p} \leq \max \left\{|x|_{p},|y|_{p}\right\} .
$$

Relative to the $p$-adic absolute value, $\mathbb{Q}$ does not satisfy the archimedean property because for each $x \in \mathbb{Q},|n x|_{p}$ will never exceed $|x|_{p}$ for any $n \in \mathbb{N}$. The completion of $\mathbb{Q}$ with respect to $|\cdot|_{p}$ is the field of $p$-adic numbers $\mathbb{Q}_{p}$. More concretely, every $z \in \mathbb{Q}_{p}$ has a unique representation

$$
z=a_{v} p^{v}+\cdots+a_{0}+a_{1} p+a_{2} p^{2}+\cdots,
$$

for some $v \in \mathbb{Z}$ and $a_{j} \in\{0,1, \ldots, p-1\}$ for all $j \geq v$ and $a_{v} \neq 0$. An important subspace of $\mathbb{Q}_{p}$ is the unit ball, $\mathbb{Z}_{p}=\left\{x \in \mathbb{Q}_{p}:|x|_{p} \leq 1\right\}$, which can also be represented as follows:

$$
\mathbb{Z}_{p}=\left\{a_{0}+a_{1} p+a_{2} p^{2}+\cdots: a_{j} \in\{0,1, \ldots, p-1\} \text { for all } j \geq 0\right\} .
$$

Using this $p$-adic expansion, one sees that

$$
\mathbb{Z}_{p}=\bigcup_{a=0}^{p-1}\left(a+p \mathbb{Z}_{p}\right)
$$

where $a+p \mathbb{Z}_{p}=\left\{y \in \mathbb{Q}_{p}:|y-a|_{p} \leq 1 / p\right\}$. Note that $\mathbb{Z}_{p}$ is compact and thus complete. Also, $\mathbb{Q}_{p}$ is a locally compact group, and hence admits a unique translation invariant Haar measure $\mu_{H}$, normalized so that $\mu_{H}\left(\mathbb{Z}_{p}\right)=1$. In particular, $\mu_{H}\left(a+p^{n} \mathbb{Z}_{p}\right)=p^{-n}$ for every $n \in \mathbb{Z}$. For general references on $p$-adic analysis, we point out, e.g., [10, 37, 38, 39.

Here and thereafter, we use the following notation: $\mathbb{N}=\{0,1,2, \ldots\}, \mathbb{N}^{*}=$ $\{1,2,3, \ldots\}$ and $\mathbb{Z}=\{0, \pm 1, \pm 2, \ldots\}$.

\section{3. $p$-Adic Fractal Strings}

Let $\Omega$ be a bounded open subset of $\mathbb{Q}_{p}$. Then it can be decomposed into a countable union of disjoint open ball $\mathbb{1}$ with radius $p^{-n_{j}}$ centered at $a_{j} \in \mathbb{Q}_{p}$,

$$
a_{j}+p^{n_{j}} \mathbb{Z}_{p}=B\left(a_{j}, p^{-n_{j}}\right)=\left\{x \in \mathbb{Q}_{p}|| x-\left.a_{j}\right|_{p} \leq p^{-n_{j}}\right\},
$$

where $n_{j} \in \mathbb{Z}$ and $j \in \mathbb{N}^{*}$. There may be many different such decompositions since each ball can always be decomposed into smaller disjoint balls [10; see Equation (2.1). However, there is a canonical decomposition of $\Omega$ into disjoint balls with respect to a suitable equivalence relation, as we now explain.

Definition 3.1. Let $U$ be an open subset of $\mathbb{Q}_{p}$. Given $x, y \in U$, we write that $x \sim y$ if and only if there is a ball $B \subseteq U$ such that $x, y \in B$.

It is easy to check that $\sim$ is an equivalence relation on $U$ (see [20]), due to the fact that either two balls are disjoint or one is contained in the other. Moreover, there are at most countably many equivalence classes since $\mathbb{Q}$ is dense in $\mathbb{Q}_{p}$.

REMARK 3.2. (Convex components) The equivalence classes of $\sim$ can be thought of as the 'convex components' of $U$. They are an appropriate substitute in the present nonarchimedean context for the notion of connected components, which is not useful in $\mathbb{Q}_{p}$ since $\mathbb{Z}_{p}$ (and hence, every interval) is totally disconnected. Note

\footnotetext{
${ }^{1}$ We shall often call a $p$-adic ball an interval. By 'ball' here, we mean a metrically closed and hence, topologically open (and closed) ball.
} 
that given any $x \in U$, the equivalence class (i.e., the convex component) of $x$ is the largest ball containing $x$ (or equivalently, centered at $x$ ) and contained in $U$.

Definition 3.3. A $p$-adic (or nonarchimedean) fractal string $\mathcal{L}_{p}$ is a bounded open subset $\Omega$ of $\mathbb{Q}_{p}$.

Thus it can be written, relative to the above equivalence relation, canonically as a disjoint union of intervals or balls:

$$
\mathcal{L}_{p}=\bigcup_{j=1}^{\infty}\left(a_{j}+p^{n_{j}} \mathbb{Z}_{p}\right)=\bigcup_{j=1}^{\infty} B\left(a_{j}, p^{-n_{j}}\right) .
$$

Here, $B\left(a_{j}, p^{-n_{j}}\right)$ is the largest ball centered at $a_{j}$ and contained in $\Omega$. We may assume that the lengths (i.e., Haar measure) of the intervals $a_{j}+p^{n_{j}} \mathbb{Z}_{p}$ are nonincreasing, by reindexing if necessary. That is,

$$
p^{-n_{1}} \geq p^{-n_{2}} \geq p^{-n_{3}} \geq \cdots>0 .
$$

REMARK 3.4. Ordinary archimedean (or real) fractal strings were introduced in 25, 26. (see also [13, 14]) and the theory of complex dimensions of those strings was developed in [28] (and its predecessors).

Definition 3.5. The geometric zeta function of a $p$-adic fractal string $\mathcal{L}_{p}$ is defined as

$$
\zeta_{\mathcal{L}_{p}}(s)=\sum_{j=1}^{\infty}\left(\mu_{H}\left(a_{j}+p^{n_{j}} \mathbb{Z}_{p}\right)\right)^{s}=\sum_{j=1}^{\infty} p^{-n_{j} s}
$$

for $\Re(s)$ sufficiently large.

REMARK 3.6. The geometric zeta function $\zeta_{\mathcal{L}_{p}}$ is well defined since the decomposition of $\mathcal{L}_{p}$ into the disjoint intervals $a_{j}+p^{n_{j}} \mathbb{Z}_{p}$ is unique. Indeed, these intervals are the equivalence classes of which the open set $\Omega$ (defining $\mathcal{L}_{p}$ ) is composed. In other words, they are the $p$-adic "convex components" (rather than the connected components) of $\Omega$. Note that in the real (or archimedean) case, there is no difference between the convex or connected components of $\Omega$, and hence the above construction would lead to the same sequence of lengths as in [28, §1.2].

As in [28, §5.3], the screen $S$ is the graph (with the vertical and horizontal axes interchanged) of a real-valued, bounded and Lipschitz continuous function $S(t)$ :

$$
S=\{S(t)+i t: t \in \mathbb{R}\} .
$$

The window $W$ is the part of the complex plane to the right of the screen $S$ (see Figure 1):

$$
W=\{s \in \mathbb{C}: \Re(s) \geq S(\Im(s))\} .
$$

Let

$$
\inf S=\inf _{t \in \mathbb{R}} S(t) \quad \text { and } \quad \sup S=\sup _{t \in \mathbb{R}} S(t),
$$

and assume that $\sup S \leq \sigma$, where $\sigma=\sigma_{\mathcal{L}_{p}}$ is the abscissa of convergence of $\mathcal{L}_{p}$ (to be precisely defined in (3.4) below).

DEFINITION 3.7. If $\zeta_{\mathcal{L}_{p}}$ has a meromorphic continuation to an open connected neighborhood of $W \subseteq \mathbb{C}$, then

$$
\mathcal{D}_{\mathcal{L}_{p}}(W)=\left\{\omega \in W: \omega \text { is a pole of } \zeta_{\mathcal{L}_{p}}\right\}
$$




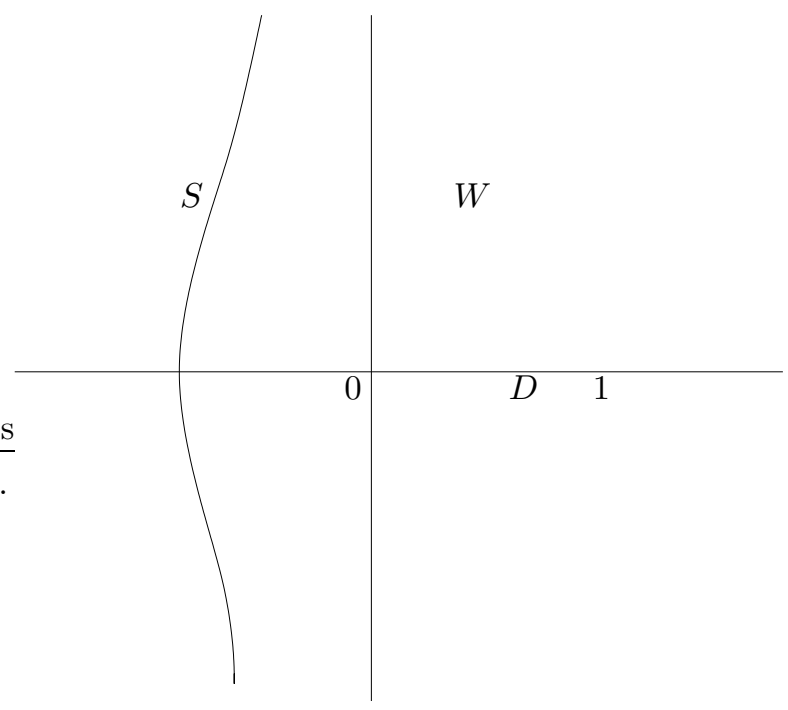

Figure 1. The screen $S$ and the window $W$.

is called the set of visible complex dimensions of $\mathcal{L}_{p}$. If no ambiguity may arise or if $W=\mathbb{C}$, we simply write $\mathcal{D}_{\mathcal{L}_{p}}=\mathcal{D}_{\mathcal{L}_{p}}(W)$ and call it the set of complex dimensions of $\mathcal{L}_{p}$.

Moreover, the abscissa of convergence of the Dirichlet series initially defining $\zeta_{\mathcal{L}_{p}}$ in Equation (3.2) is denoted by $\sigma=\sigma_{\mathcal{L}_{p}}$. Recall that it is defined by

$$
\sigma_{\mathcal{L}_{p}}=\inf \left\{\alpha \in \mathbb{R}: \sum_{j=1}^{\infty} p^{-n_{j} \alpha}<\infty\right\} .
$$

REMARK 3.8. In particular, if $\zeta_{\mathcal{L}_{p}}$ is entire, which occurs only in the trivial case when $\mathcal{L}_{p}$ is given by a finite union of intervals, then $\sigma_{\mathcal{L}_{p}}=-\infty$. Otherwise, $\sigma_{\mathcal{L}_{p}} \geq 0$ since $\mathcal{L}_{p}$ is composed of infinitely many intervals, and hence $\zeta_{\mathcal{L}_{p}}(0)=\infty$. Moreover, $\sigma_{\mathcal{L}_{p}}<\infty$ since $\sigma_{\mathcal{L}_{p}} \leq D_{M} \leq 1$, where $D_{M}$ is the Minkowski dimension of $\mathcal{L}_{p}$. (The fact that $D_{M} \leq 1$ follows since the Haar measure of $\Omega$ is finite and coincides with $\zeta_{\mathcal{L}_{p}}(1)$.) Indeed, as is shown in [20, we actually have $\sigma_{\mathcal{L}_{p}}=D_{M}$ for any nontrivial $p$-adic fractal string. This is the case, for example, for the 3-adic Cantor string introduced in [17, for which $\sigma_{\mathcal{L}_{p}}=D_{M}=\log _{3} 2$; see Example 3.9 below.

Observe that since $\mathcal{D}_{\mathcal{L}_{p}}(W)$ is defined as a subset of the poles of a meromorphic function, it is at most countable.

Finally, we note that it is well known that $\zeta_{\mathcal{L}_{p}}$ is holomorphic for $\Re(s)>\sigma_{\mathcal{L}_{p}}$; see, e.g., [39]. Hence,

$$
\mathcal{D}_{\mathcal{L}_{p}} \subset\left\{s \in \mathbb{C}: \Re(s) \leq \sigma_{\mathcal{L}_{p}}\right\} .
$$

EXAmple 3.9. The 3 -adic Cantor string is given by

$$
\mathcal{C S}_{3}=\left(1+3 \mathbb{Z}_{3}\right) \cup\left(3+9 \mathbb{Z}_{3}\right) \cup\left(5+9 \mathbb{Z}_{3}\right) \cup \cdots
$$


By definition, the geometric zeta function of $\mathcal{C S}_{3}$ is given by

$$
\begin{aligned}
\zeta_{\mathcal{C S}_{3}}(s) & =\left(\mu_{H}\left(1+3 \mathbb{Z}_{3}\right)\right)^{s}+\left(\mu_{H}(3+9 \mathbb{Z})\right)^{s}+\left(\mu_{H}\left(5+9 \mathbb{Z}_{3}\right)\right)^{s}+\cdots \\
& =\sum_{v=1}^{\infty} \frac{2^{v-1}}{3^{v s}}=\frac{3^{-s}}{1-2 \cdot 3^{-s}} \text { for } \Re(s)>\log _{3} 2 .
\end{aligned}
$$

Hence, by analytic continuation, the meromorphic extension of $\zeta_{\mathcal{C S}_{3}}$ to the entire complex plane $\mathbb{C}$ exists and is given by

$$
\zeta_{\mathcal{C S}_{3}}(s)=\frac{3^{-s}}{1-2 \cdot 3^{-s}}, \quad \text { for } s \in \mathbb{C},
$$

with poles at

$$
\omega=\frac{\log 2}{\log 3}+i n \frac{2 \pi}{\log 3}, \quad n \in \mathbb{Z} .
$$

Therefore, the set of complex dimensions of $\mathcal{C S}_{3}$ is given by

$$
\mathcal{D}_{\mathcal{C S}_{3}}=\{D+i n \mathbf{p}: n \in \mathbb{Z}\}
$$

where $D=\log _{3} 2$ is the dimension of $\mathcal{C S}_{3}$ and $\mathbf{p}=2 \pi / \log 3$ is its oscillatory period. Moreover, the residue of $\zeta_{\mathcal{C S}_{3}}(s)$ at $s=D+i n \mathbf{p}$ is given by

$$
\operatorname{res}\left(\zeta_{\mathcal{C S}} ; D+i n \mathbf{p}\right)=\frac{1}{2 \log 3}
$$

independently of $n \in \mathbb{Z}$. Finally, note that $\zeta_{\mathcal{C} \mathcal{S}_{3}}$ is a rational function of $z:=3^{-s}$, i.e.,

$$
\zeta_{\mathcal{C S}_{3}}(s)=\frac{z}{1-2 z}
$$

The geometric zeta function $\zeta_{\mathcal{C S}}$ in Equation (3.6) is bounded in the left halfplane $\{s \in \mathbb{C}: \Re(s) \leq 0\}$. In general, the geometric zeta function of a real or $p$-adic self-similar fractal string is always strongly languid, i.e.,

- There exist constants $A, C>0$ such that for all $t \in \mathbb{R}$ and $m \gg 0$,

$$
\left|\zeta_{\mathcal{L}_{p}}(-m+i t)\right| \leq C A^{|t|} .
$$

See [28, $\S 5.3]$ or $\mathbf{2 0}$ for the general definition of "languid".

\section{Volume of Inner Tubes}

In this section, based on a part of [20, we provide a suitable analog in the $p$-adic case of the 'boundary' of a fractal string and of the associated inner tubes (inner $\varepsilon$-neighborhoods). Moreover, we give the $p$-adic counterpart of the expression that yields the volume of the inner tubes (see Theorem 4.3). This result serves as a starting point in $\mathbf{2 0}$ for proving the corresponding explicit tube formula.

Definition 4.1. Given a point $a \in \mathbb{Q}_{p}$ and a positive real number $r>0$, let $B=B(a, r)=\left\{x \in \mathbb{Q}_{p}:|x-a|_{p} \leq r\right\}$ be a metrically closed ball in $\mathbb{Q}_{p}$, as above2 We call $S=S(a, r)=\left\{x \in \mathbb{Q}_{p}:|x-a|_{p}=r\right\}$ the sphere of $B$

\footnotetext{
${ }^{2}$ Recall that it follows from the ultrametricity of $|\cdot|_{p}$ that $B$ is topologically both closed and open (i.e., clopen) in $\mathbb{Q}_{p}$.

${ }^{3}$ In our sense, $S$ also coincides with the 'metric boundary' of $B$, as given in this definition.
} 
Let $\mathcal{L}_{p}=\bigcup_{j=1}^{\infty} B\left(a_{j}, r_{j}\right)$ be a $p$-adic fractal string. We then define the metric boundary $\beta \mathcal{L}_{p}$ of $\mathcal{L}_{p}$ to be the disjoint union of the corresponding spheres, i.e.,

$$
\beta \mathcal{L}_{p}=\bigcup_{j=1}^{\infty} S\left(a_{j}, r_{j}\right)
$$

Given $\varepsilon>0$, define the thick p-adic 'inner $\varepsilon$-neighborhood' of $\mathcal{L}_{p}$ to be

$$
\mathcal{N}_{\varepsilon}=\mathcal{N}_{\varepsilon}\left(\mathcal{L}_{p}\right):=\left\{x \in \mathcal{L}_{p}: d_{p}\left(x, \beta \mathcal{L}_{p}\right)<\varepsilon\right\},
$$

where $d_{p}(x, E)=\inf \left\{|x-y|_{p}: y \in E\right\}$ is the $p$-adic distance of $x \in \mathbb{Q}_{p}$ to a subset $E \subset \mathbb{Q}_{p}$. Then the volume $\mathcal{V}_{\mathcal{L}_{p}}(\varepsilon)$ of the thick inner $\varepsilon$-neighborhood of $\mathcal{L}_{p}$ is defined to be the Haar measure of $\mathcal{N}_{\varepsilon}$, i.e., $\mathcal{V}_{\mathcal{L}_{p}}(\varepsilon)=\mu_{H}\left(\mathcal{N}_{\varepsilon}\right)$.

Recall that $\zeta_{\mathcal{L}_{p}}(1)=\sum_{j=1}^{\infty} p^{-n_{j}}$ is the volume of $\mathcal{L}_{p}$ (or rather, of the bounded open subset $\Omega$ of $\mathbb{Q}_{p}$ representing $\left.\mathcal{L}_{p}\right): \zeta_{\mathcal{L}_{p}}(1)=\mu_{H}\left(\mathcal{L}_{p}\right)=\mu_{H}(\Omega)<\infty$.

Definition 4.2. Given $\varepsilon>0$, the p-adic 'inner $\varepsilon$-neighborhood' (or 'inner tube') of $\mathcal{L}_{p}$ is given by

$$
N_{\varepsilon}=N_{\varepsilon}\left(\mathcal{L}_{p}\right):=\mathcal{N}_{\varepsilon} \backslash \beta \mathcal{L}_{p} .
$$

Then the volume $V_{\mathcal{L}_{p}}(\varepsilon)$ of the inner $\varepsilon$-neighborhood of $\mathcal{L}_{p}$ is defined to be the Haar measure of $N_{\varepsilon}$, i.e.,

$$
V_{\mathcal{L}_{p}}(\varepsilon):=\mu_{H}\left(N_{\varepsilon}\right)=\mathcal{V}_{\mathcal{L}_{p}}(\varepsilon)-\mu_{H}\left(\beta \mathcal{L}_{p}\right) .
$$

We next state the nonarchimedean counterpart of [25. Eq. (3.2)] (see also 28, Eq. (8.1)]), which is the key result in 20 that will enable us to obtain an appropriate $p$-adic analog of the fractal tube formula as well as of the notion of Minkowski dimension and content (see $\$ 9$ and $\$ 10$ ).

Theorem 4.3 (Volume of inner tubes). Let $\mathcal{L}_{p}=\bigcup_{j=1}^{\infty} B\left(a_{j}, p^{-n_{j}}\right)$ be a p-adic fractal string. Then, for any $\varepsilon>0$, we have

$$
V_{\mathcal{L}_{p}}(\varepsilon)=p^{-1}\left(\zeta_{\mathcal{L}_{p}}(1)-\sum_{j=1}^{k} p^{-n_{j}}\right),
$$

where $k=k(\varepsilon)$ is the largest integer such that $n_{k} \leq \log _{p} \varepsilon^{-1}$.

Remark 4.4. Note that $\lim _{\varepsilon \rightarrow 0^{+}} V_{\mathcal{L}_{p}}(\varepsilon)=0$, which justifies Definition 4.2, see 20. Further observe that even though 'the' metric boundary may depend on the choice of the centers $a_{j}\left(j \in \mathbb{N}^{*}\right)$, both $\mathcal{V}_{\mathcal{L}_{p}}(\varepsilon)$ and $V_{\mathcal{L}_{p}}(\varepsilon)$ are indepedent of this choice (in light of Equation (4.4)).

EXAmPLE 4.5 (The explicit tube formula for 3 -adic Cantor string). Let $\varepsilon>0$. Then, by Theorem 4.3 , we have

$$
V_{\mathcal{C S}_{3}}(\varepsilon)=\frac{1}{3} \sum_{n=k+1}^{\infty} \frac{2^{n-1}}{3^{n}}=\frac{1}{3}\left(\frac{2}{3}\right)^{k},
$$

where $k:=\left[\log _{3} \varepsilon^{-1}\right]$. Let $x:=\log _{3} \varepsilon^{-1}=k+\{x\}$, where $\{x\}$ is the fractional part of $x$. Then a simple computation shows that $\left(\frac{2}{3}\right)^{x}=\varepsilon^{1-D}$ and $e^{2 \pi i n x}=\varepsilon^{-i n \mathbf{p}}$, with $D=\log _{3} 2$ and $\mathbf{p}=2 \pi / \log 3$ as in Example 3.9. Using the Fourier expansion for the periodic function $b^{-\{x\}}$, as given by [28 Eq. (1.13)], for $b=3^{-1}$ and the above 
value of $x$, we obtain an expansion in terms of the complex dimensions $\omega=D+i n \mathbf{p}$ of $\mathcal{C S}_{3}$ :

$$
\begin{aligned}
V_{\mathcal{C S}_{3}}(\varepsilon) & =\frac{3^{-1}}{2 \log 3} \sum_{n \in \mathbb{Z}} \frac{\varepsilon^{1-D-i n \mathbf{p}}}{1-D-i n \mathbf{p}} \\
& =\frac{1}{6 \log 3} \sum_{\omega \in \mathcal{D}_{\mathcal{C S}}} \frac{\varepsilon^{1-\omega}}{1-\omega}
\end{aligned}
$$

since $\mathcal{D}_{\mathcal{C S}_{3}}$ is given by (3.7).

\section{Explicit Tube Formulas for $p$-Adic Fractal Strings}

The following result is the counterpart in this context of Theorem 8.1 of $[\mathbf{2 8}$, the distributional tube formula for real fractal strings. It is established in [20] by using, in particular, the extended distributional explicit formula of $\mathbf{2 8}$, Thms. 5.26 and 5.27], along with the expression for the volume of thin inner $\varepsilon$-tubes stated in Theorem 4.3 .

TheOREM 5.1. Let $\mathcal{L}_{p}$ be a languid p-adic fractal string. Further assume that $\left.\sigma_{\mathcal{L}_{p}}<1\right]$ Then the volume of the thin inner $\varepsilon$-neighborhood of $\mathcal{L}_{p}$ is given by

$$
V_{\mathcal{L}_{p}}(\varepsilon)=\sum_{\omega \in \mathcal{D}_{\mathcal{L}_{p}}(W)} \operatorname{res}\left(\frac{p^{-1} \zeta_{\mathcal{L}_{p}}(s) \varepsilon^{1-s}}{1-s} ; \omega\right)+\mathcal{R}_{p}(\varepsilon),
$$

where $\mathcal{D}_{\mathcal{L}_{p}}(W)$ is the set of visible complex dimensions of $\mathcal{L}_{p}$. Here, the distributional error term is given by

$$
\mathcal{R}_{p}(\varepsilon)=\frac{1}{2 \pi i} \int_{S} \frac{p^{-1} \zeta_{\mathcal{L}_{p}}(s) \varepsilon^{1-s}}{1-s} d s
$$

and is estimated distributionally $y^{5}$ by

$$
\mathcal{R}_{p}(\varepsilon)=O\left(\varepsilon^{1-\sup S}\right), \quad \text { as } \varepsilon \rightarrow 0^{+} .
$$

Moreover, if $\mathcal{L}_{p}$ is strongly languid (which is the case of all p-adic self-similar strings; see $\S 3$ and $\S 9)$, then we can take $W=\mathbb{C}$ and $\mathcal{R}_{p}(\varepsilon) \equiv 0$.

Corollary 5.2. If, in addition to the hypotheses in Theorem 5.1, we assume that all the visible complex dimensions of $\mathcal{L}_{p}$ are simple, then

$$
V_{\mathcal{L}_{p}}(\varepsilon)=\sum_{\omega \in \mathcal{D}_{\mathcal{L}_{p}}(W)} c_{\omega} \frac{\varepsilon^{1-\omega}}{1-\omega}+\mathcal{R}_{p}(\varepsilon),
$$

where $c_{\omega}=p^{-1} \operatorname{res}\left(\zeta_{\mathcal{L}_{p}} ; \omega\right)$. Here, the error term $\mathcal{R}_{p}$ is given by (5.2) and is estimated by (5.3) in the languid case. Furthermore, we have $\mathcal{R}_{p}(\varepsilon) \equiv 0$ in the strongly languid case (yielding an exact tube formula), provided we choose $W=\mathbb{C}$.

\footnotetext{
${ }^{4}$ Recall from Remark 3.8 that we always have $\sigma_{\mathcal{L}_{p}} \leq 1$. Moreover, if $\mathcal{L}_{p}$ is self-similar, then $\sigma_{\mathcal{L}_{p}}<1$ (in light of $3 \mathbf{3 5}$ and the definition of $\sigma_{\mathcal{L}_{p}}$ ).

${ }^{5}$ As in 28 Defn. 5.29].
} 
Remark 5.3. In [28, Ch. 8], under different sets of assumptions, both distributional and pointwise tube formulas are obtained for archimedean fractal strings (and also, for archimedean self-similar fractal strings). (See, in particular, Theorems 8.1 and 8.7, along with $\S 8.4$ in $\mathbf{2 8}$.) At least for now, in the nonarchimedean case, we limit ourselves to discussing distributional explicit tube formulas. We expect, however, that under appropriate hypotheses, one should be able to obtain a pointwise fractal tube formula for $p$-adic fractal strings and especially, for $p$-adic self-similar strings. In fact, for the simple examples of the nonarchimedean Cantor and Fibonacci strings, the direct derivation of the fractal tube formula (5.4) yields a formula that is valid pointwise and not just distributionally. (See, in particular, Examples 4.5 and 10.7) We leave the consideration of such possible extensions to a future work.

EXAmPle 5.4 (The explicit tube formula for 3-adic Cantor string revisited). By Equation (3.8), we have that

$$
\operatorname{res}\left(\zeta_{\mathcal{C S}} ; \omega\right)=\frac{1}{2 \log 3}
$$

independently of $\omega \in \mathcal{D}_{\mathcal{C S}_{3}}$. So, using the last part of Theorem [5.1, the exact fractal tube formula for the 3 -adic Cantor string is found to be

$$
V_{\mathcal{C S}_{3}}(\varepsilon)=\frac{3^{-1}}{2 \log 3} \sum_{\omega \in \mathcal{D}_{\mathcal{C S}}} \frac{\varepsilon^{1-\omega}}{1-\omega}
$$

which is exactly the same as Equation (4.6).

Note that since $\mathcal{C S}_{3}$ has simple complex dimensions, we may also apply Corollary 5.2 (in the strongly languid case when $W=\mathbb{C}$ ) in order to precisely recover Equation (5.5). (Alternatively, we could use Corollary 9.2 in 99 below.)

We may rewrite (4.6) or (5.5) in the following form (which agrees with the tube formula to be obtained in Corollary 9.2 ):

$$
V_{\mathcal{C S}}(\varepsilon)=\varepsilon^{1-D} G_{\mathcal{C S}}\left(\log _{3} \varepsilon^{-1}\right),
$$

where $G_{\mathcal{C S}_{3}}$ is the nonconstant periodic function of period 1 on $\mathbb{R}$ given by

$$
G_{\mathcal{C S}_{3}}(x):=\frac{1}{6 \log 3} \sum_{n \in \mathbb{Z}} \frac{e^{2 \pi i n x}}{1-D-i n \mathbf{p}} .
$$

Finally, we note that since the Fourier series

$$
\sum_{n \in \mathbb{Z}} \frac{e^{2 \pi i n x}}{1-D-i n \mathbf{p}}
$$

is pointwise convergent on $\mathbb{R}$, the above direct computation of $V_{\mathcal{C S}}(\varepsilon)$ shows that (4.6) and (5.5) actually hold pointwise rather than distributionally.

\section{Nonarchimedean Self-Similar Strings}

Nonarchimedean (or $p$-adic) self-similar strings form an important class of $p$ adic fractal strings. In this section, we first recall the construction of these strings, as provided in 18 and [19; see $\$ 6.1$, Later on, we will give an explicit expression for their geometric zeta functions and deduce from it the periodic structure of their poles (or complex dimensions) and zeros, as obtained in [18; see 978.1 . Moreover, in 99 , we will deduce from the results of $\$ 5$ and $\$ 7,8$ the special form of the fractal 
tube formula for $p$-adic self-similar strings. Finally, in $\$ 10$, we will apply this latter result in order to calculate the average Minkowski content of such strings.

6.1. Geometric Construction. Before explaining how to construct arbitrary $p$-adic self-similar strings, we need to introduce a definition and a few facts pertaining to $p$-adic similarity transformations 6

Definition 6.1. A map $\Phi: \mathbb{Z}_{p} \longrightarrow \mathbb{Z}_{p}$ is called a similarity contraction mapping of $\mathbb{Z}_{p}$ if there is a real number $r \in(0,1)$ such that

$$
|\Phi(x)-\Phi(y)|_{p}=r \cdot|x-y|_{p},
$$

for all $x, y \in \mathbb{Z}_{p}$.

Unlike in Euclidean space (and in the real line $\mathbb{R}$, in particular), it is not true that every similarity transformation of $\mathbb{Q}_{p}$ (or of $\mathbb{Z}_{p}$ ) is necessarily affine. Actually, in the nonarchimedean world (for example, in $\mathbb{Q}_{p}^{d}$, with $d \geq 1$ ), and in the $p$-adic line $\mathbb{Q}_{p}$, in particular, there are a lot of similarities which are not affine. However, it is known (see, e.g., [38) that every analytic similarity must be affine 7 Hence, from now on, we will be working with a similarity contraction mapping $\Phi: \mathbb{Z}_{p} \longrightarrow \mathbb{Z}_{p}$ that is affine. Thus we assume that there exist constants $a, b \in \mathbb{Z}_{p}$ with $|a|_{p}<1$ such that $\Phi(x)=a x+b$ for all $x \in \mathbb{Z}_{p}$. Regarding the scaling factor $a$ of the contraction, it is well known that it can be written as $a=u \cdot p^{n}$, for some unit $u \in \mathbb{Z}_{p}$ (i.e., $|u|_{p}=1$ ) and $n \in \mathbb{N}^{*}$ (see [30]). Then $r=|a|_{p}=p^{-n}$. We summarize this fact in the following lemma:

LEMma 6.2. Let $\Phi(x)=a x+b$ be an affine similarity contraction mapping of $\mathbb{Z}_{p}$ with the scaling ratio $r$. Then $b \in \mathbb{Z}_{p}$ and $a \in p \mathbb{Z}_{p}$, and the scaling factor is $r=|a|_{p}=p^{-n}$ for some $n \in \mathbb{N}^{*}$.

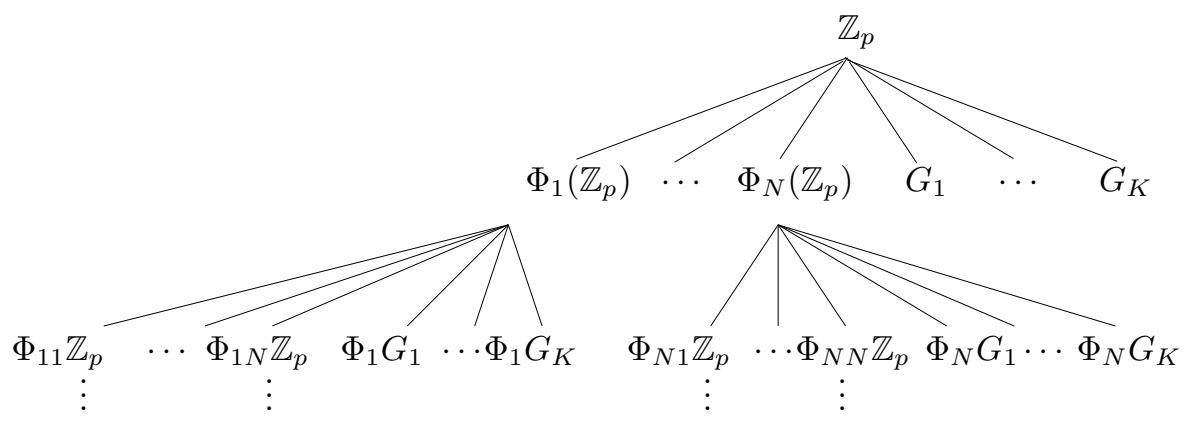

Figure 2. Construction of a $p$-adic self-similar fractal string.

For simplicity, let us take the unit interval (or ball) $\mathbb{Z}_{p}$ in $\mathbb{Q}_{p}$ and construct a $p$-adic (or nonarchimedean) self-similar string $\mathcal{L}_{p}$ as follows (see [18]).8 Let

\footnotetext{
${ }^{6}$ The standard definition of self-similarity (in Euclidean space or in more general complete metric spaces) can be found in [9] and in [5, for example.

${ }^{7}$ Here, a map $f: \mathbb{Q}_{p} \longrightarrow \mathbb{Q}_{p}$ is said to be analytic if it admits a convergent power series expansion about 0 , and with coefficients in $\mathbb{Q}_{p}$, that is convergent in all of $\mathbb{Q}_{p}$.

${ }^{8}$ In the sequel, $\mathcal{L}_{p}$ is interchangeably called a $p$-adic or nonarchimedean self-similar string.
} 
$N \geq 2$ be an integer and $\Phi_{1}, \ldots, \Phi_{N}: \mathbb{Z}_{p} \longrightarrow \mathbb{Z}_{p}$ be $N$ affine similarity contraction mappings with the respective scaling ratios $r_{1}, \ldots, r_{N} \in(0,1)$ satisfying

$$
1>r_{1} \geq r_{2} \geq \cdots \geq r_{N}>0
$$

see Figure 2. Assume that

$$
\sum_{j=1}^{N} r_{j}<1
$$

and the images $\Phi_{j}\left(\mathbb{Z}_{p}\right)$ of $\mathbb{Z}_{p}$ do not overlap, i.e., $\Phi_{j}\left(\mathbb{Z}_{p}\right) \cap \Phi_{l}\left(\mathbb{Z}_{p}\right)=\emptyset$ for all $j \neq l$. Note that it follows from Equation (6.2) that $\bigcup_{j=1}^{N} \Phi_{j}\left(\mathbb{Z}_{p}\right)$ is not all of $\mathbb{Z}_{p}$. We therefore have the following (nontrivial) decomposition of $\mathbb{Z}_{p}$ into disjoint $p$-adic intervals:

$$
\mathbb{Z}_{p}=\bigcup_{j=1}^{N} \Phi_{j}\left(\mathbb{Z}_{p}\right) \cup \bigcup_{k=1}^{K} G_{k},
$$

where $G_{k}$ is defined below.

In a procedure reminiscent of the construction of the ternary Cantor set, we then subdivide the interval $\mathbb{Z}_{p}$ by means of the subintervals $\Phi_{j}\left(\mathbb{Z}_{p}\right)$. Then the convex 9 components of

$$
\mathbb{Z}_{p} \backslash \bigcup_{j=1}^{N} \Phi_{j}\left(\mathbb{Z}_{p}\right)
$$

are the first substrings of the $p$-adic self-similal string $\mathcal{L}_{p}$, say $G_{1}, G_{2}, \ldots, G_{K}$, with $K \geq 1$. These intervals $G_{k}$ are called the generators, the deleted intervals in the first generation of the construction of $\mathcal{L}_{p} 10$ The length of each $G_{k}$ is denoted by $g_{k}$; so that $g_{k}=\mu_{H}\left(G_{k}\right) 11$ Without loss of generality, we may assume that the lengths $g_{1}, g_{2}, \ldots, g_{K}$ of the first substrings (i.e., intervals) of $\mathcal{L}_{p}$ satisfy

$$
1>g_{1} \geq g_{2} \geq \cdots \geq g_{K}>0 .
$$

It follows from Equation (6.3) and the additivity of Haar measure $\mu_{H}$ that

$$
\sum_{j=1}^{N} r_{j}+\sum_{k=1}^{K} g_{k}=1
$$

We then repeat this process with each of the remaining subintervals $\Phi_{j}\left(\mathbb{Z}_{p}\right)$ of $\mathbb{Z}_{p}$, for $j=1,2, \ldots, N$. And so on, ad infinitum. As a result, we obtain a $p$-adic self-similar string $\mathcal{L}_{p}=l_{1}, l_{2}, l_{3}, \ldots$, consisting of intervals of length $l_{n}$ given by

$$
r_{\nu_{1}} r_{\nu_{2}} \cdots r_{\nu_{q}} g_{k}
$$

for $k=1, \ldots, K$ and all choices of $q \in \mathbb{N}$ and $\nu_{1}, \ldots, \nu_{q} \in\{1, \ldots, N\}$. Thus, the lengths are of the form $r_{1}^{e_{1}} \ldots r_{N}^{e_{N}} g_{k}$ with $e_{1}, \ldots, e_{N} \in \mathbb{N}$ (but not all zero).

\footnotetext{
${ }^{9}$ We choose the convex components instead of the connected components because $\mathbb{Z}_{p}$ is totally disconnected. Naturally, no such distinction is necessary in the archimedean case; cf. [28. §2.1.1]. Here and elsewhere in this paper, a subset $E$ of $\mathbb{Q}_{p}$ is said to be 'convex' if for every $x, y \in E$, the $p$-adic segment $\left\{t x+(1-t) y: t \in \mathbb{Z}_{p}\right\}$ lies entirely in $E$.

${ }^{10}$ Their archimedean counterparts are called 'gaps' in [28 Ch. 2 and $\left.\S 8.4\right]$, where archimedean self-similar strings are introduced.

${ }^{11}$ We note that the lengths $g_{k}(k=1,2, \ldots, K)$ will sometimes be called the (nonarchimedean) 'gaps' or 'gap sizes' in the sequel.
} 
In [18, the classic notion of self-similarity is extended to the nonarchimedean setting, much as in 9, where the underlying complete metric space is allowed to be arbitrary. We note that the next result follows by applying the classic Contraction Mapping Principle to the complete metric space of all nonempty compact subsets of $\mathbb{Z}_{p}$. (Note that $\mathbb{Z}_{p}$ itself is complete since it is a compact metric space.)

THEOREM 6.3. There is a unique nonempty compact subset $\mathcal{S}_{p}$ of $\mathbb{Z}_{p}$ such that

$$
\mathcal{S}_{p}=\bigcup_{j=1}^{N} \Phi_{j}\left(\mathcal{S}_{p}\right) .
$$

The set $\mathcal{S}_{p}$ is called the $p$-adic self-similar set associated with the self-similar system $\boldsymbol{\Phi}=\left\{\Phi_{1}, \ldots, \Phi_{N}\right\}$. It is also called the $\boldsymbol{\Phi}$-invariant set.

The relationship between the $p$-adic self-similar string $\mathcal{L}_{p}$ and the above $p$-adic self-similar set $\mathcal{S}_{p}$ is given by the following theorem, also obtained in [18] 12

TheOREM 6.4. (i) $\mathcal{L}_{p}=\mathbb{Z}_{p} \backslash \mathcal{S}_{p}$, the complement of $\mathcal{S}_{p}$ in $\mathbb{Z}_{p}$.

(ii) $\mathcal{L}_{p}=\bigcup_{\alpha=0}^{\infty} \bigcup_{w \in W_{\alpha}} \bigcup_{k=1}^{K} \Phi_{w}\left(G_{k}\right)$, while $\mathcal{S}_{p}=\bigcap_{\alpha=0}^{\infty} \bigcup_{w \in W_{\alpha}} \Phi_{w}\left(\mathbb{Z}_{p}\right)$, where $W_{\alpha}=\{1,2, \ldots, N\}^{\alpha}$ denotes the set of all finite words on $N$ symbols, of length $\alpha$, and $\Phi_{w}:=\Phi_{w_{\alpha}} \circ \cdots \circ \Phi_{w_{1}}$ for $w=\left(w_{1}, \ldots, w_{\alpha}\right) \in W_{\alpha}$.

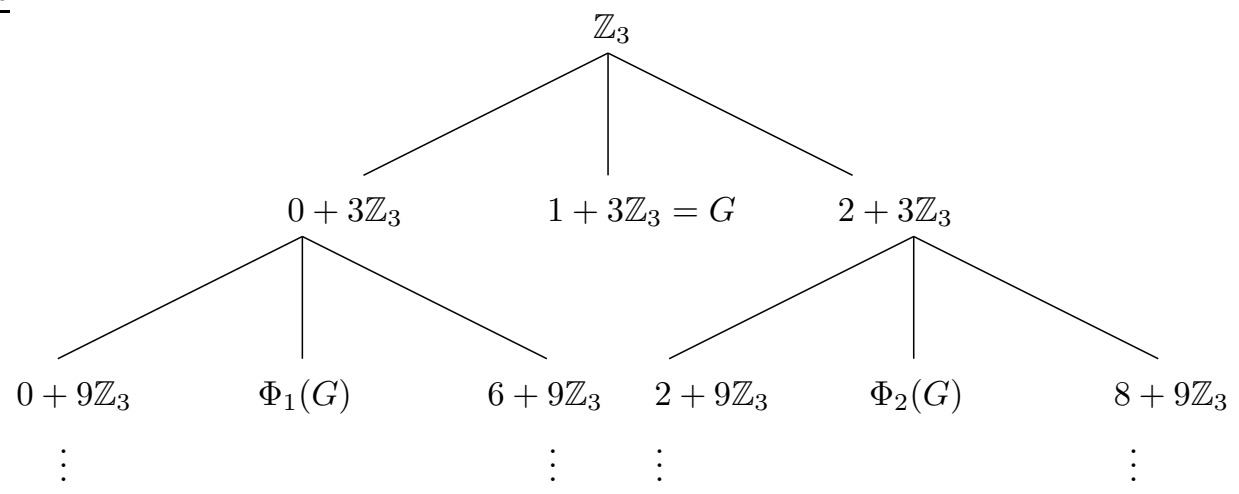

Figure 3. Construction of the 3-adic Cantor string $\mathcal{C S}_{3}$ via an IFS.

ExAmPle 6.5 (Nonarchimedean Cantor string as a 3-adic self-similar string). In this example, we review the construction of the nonarchimedean Cantor string $\mathcal{C S}_{3}$, as introduced in 17 and revisited in [18. Our main point here is to stress the fact that $\mathcal{C S}_{3}$ is a special case of a $p$-adic self-similar string, as constructed just above, and to prepare the reader for more general results about nonarchimedean self-similar strings, as obtained in the rest of this paper.

Let $\Phi_{1}, \Phi_{2}: \mathbb{Z}_{3} \longrightarrow \mathbb{Z}_{3}$ be the two affine similarity contraction mappings of $\mathbb{Z}_{3}$ given by

$$
\Phi_{1}(x)=3 x \quad \text { and } \quad \Phi_{2}(x)=2+3 x
$$

\footnotetext{
${ }^{12}$ In Theorem 6.4 $\mathcal{L}_{p}$ is not viewed as a sequence of lengths but is viewed instead as the open set which is canonically given by a disjoint union of intervals (its $p$-adic convex components), as described in the above construction of a $p$-adic self-similar string.
} 
with the same scaling ratio $r=3^{-1}$ (i.e., $r_{1}=r_{2}=3^{-1}$ ). By analogy with the construction of the real Cantor string, subdivide the interval $\mathbb{Z}_{3}$ into subintervals

$$
\Phi_{1}\left(\mathbb{Z}_{3}\right)=0+3 \mathbb{Z}_{3} \quad \text { and } \quad \Phi_{2}\left(\mathbb{Z}_{3}\right)=2+3 \mathbb{Z}_{3} .
$$

The remaining (3-adic) convex component

$$
\mathbb{Z}_{3} \backslash \bigcup_{j=1}^{2} \Phi_{j}\left(\mathbb{Z}_{3}\right)=1+3 \mathbb{Z}_{3}=G
$$

is the first substring of a 3 -adic self-similar string, called the nonarchimedean Cantor string and denoted by $\mathcal{C S}_{3}$ [17. The length of $G$ is $l_{1}=\mu_{H}\left(1+3 \mathbb{Z}_{p}\right)=3^{-1}$. By repeating this process with the remaining subintervals $\Phi_{j}\left(\mathbb{Z}_{3}\right)$, for $j=1,2$, and continuing on, ad infinitum, we eventually obtain a sequence $\mathcal{C S}_{3}=l_{1}, l_{2}, l_{3}, \ldots$, associated with the open set resulting from this construction and consisting of intervals of lengths $l_{v}=3^{-v}$ with multiplicities $m_{v}=2^{v-1}$, for $v \in \mathbb{N}^{*}$. As follows from this construction (see Figure 3 and Equation (6.7), along with part (ii) of Theorem 6.4), the nonarchimedean Cantor string $\mathcal{C S}_{3}$ can also be written as

$$
\mathcal{C S}_{3}=\left(1+3 \mathbb{Z}_{3}\right) \cup\left(3+9 \mathbb{Z}_{3}\right) \cup\left(5+9 \mathbb{Z}_{3}\right) \cup \cdots .
$$

We refer the interested reader to $\mathbf{1 7}$ and $1 \mathbf{1 9}$ for additional information concerning the nonarchimedean Cantor string $\mathcal{C S}_{3}$ and the associated nonarchimedean Cantor set $\mathcal{C}_{3}$. We just mention here that in light of part (i) of Theorem 6.4 we can recover the 3 -adic Cantor set $\mathcal{C}_{3}$ as the complement of the 3 -adic Cantor string $\mathcal{C S}_{3}$ in the unit interval (and vice-versa):

$$
\mathcal{C S}_{3}=\mathbb{Z}_{3} \backslash \mathcal{C}_{3}, \quad \text { and so } \quad \mathcal{C}_{3}=\mathbb{Z}_{3} \backslash \mathcal{C} \mathcal{S}_{3} .
$$

Indeed, according to Theorem 6.3, $\mathcal{C}_{3}$ is the self-similar set associated with the self-similar system $\boldsymbol{\Phi}=\left\{\Phi_{1}, \Phi_{2}\right\}$.

\section{Geometric Zeta Function of $p$-Adic Self-Similar Strings}

In this section, as well as in $₫ 8$ and 98.1 , we will survey results obtained in $[\mathbf{1 8}$ about the geometric zeta functions and the complex dimensions of $p$-adic self-similar strings. (See also [19, where the archimedean and nonarchimedean situations are contrasted.)

In the next theorem, we provide a first expression for the geometric zeta function of a nonarchimedean self-similar string. At first sight, this expression is almost identical to the one obtained in the archimedean case in [28, Thm. 2.4]. Later on, however, we will see that unlike in the archimedean case where the situation is considerably more subtle and complicated (cf. 28, Thms. 2.17 and 3.6]), this expression can be significantly simplified since the two potentially transcendental functions appearing in the denominator and numerator of Equation (7.1) below can always be made rational; see Theorem 8.1 in 88 .

THEOREM 7.1. Let $\mathcal{L}_{p}$ be a p-adic self-similar string with scaling ratios $\left\{r_{j}\right\}_{j=1}^{N}$ and gaps $\left\{g_{k}\right\}_{k=1}^{K}$, as in the above construction. Then the geometric zeta function of $\mathcal{L}_{p}$ has a meromorphic extension to the whole complex plane $\mathbb{C}$ and is given by

$$
\zeta_{\mathcal{L}_{p}}(s)=\frac{\sum_{k=1}^{K} g_{k}^{s}}{1-\sum_{j=1}^{N} r_{j}^{s}}, \quad \text { for } \quad s \in \mathbb{C} .
$$


COROLlary 7.2. The set of complex dimensions of a p-adic self-similar fractal string $\mathcal{L}_{p}$ is contained in the set of complex solutions $\omega$ of the Moran equation $\sum_{j=1}^{N} r_{j}^{\omega}=1$. If the string has a single generator $(i . e .$, if $K=1$ ), then this inclusion is an equality 13

Definition 7.3. A $p$-adic self-similar string $\mathcal{L}_{p}$ is said to be lattice if the multiplicative group generated by the scaling ratios $r_{1}, r_{2}, \ldots, r_{N}$ is discrete in $(0, \infty)$. Otherwise, $\mathcal{L}_{p}$ is said to be nonlattice. Furthermore, $\mathcal{L}_{p}$ is said to be strongly lattice if the multiplicative group generated by $\left\{r_{1}, \ldots, r_{N}, g_{1}, \ldots, g_{K}\right\}$ is discrete in $(0, \infty)$. Naturally, a strongly lattice string is also a lattice string.

THEOREM 7.4. Every p-adic self-similar fractal string is strongly lattice.

REMARK 7.5. Theorem 7.4 follows from the fact that all the scaling ratios $r_{j}$ and the gaps $g_{k}$ must belong to the group $p^{\mathbb{Z}}$, as will be discussed below in more detail in $\$ 8$ It follows that $p$-adic self-similar strings are lattice strings in a very strong sense, namely, their geometric zeta functions are rational functions of a suitable variable $z$ (see Theorem 8.1 below).

REMARK 7.6. Theorem 7.4 is in sharp contrast with the usual theory of real selfsimilar strings developed in [28, Chs. 2 and 3]. Indeed, there are both lattice and nonlattice strings in the archimedean case. Furthermore, generically, archimedean self-similar strings are nonlattice. Moreover, it is shown in [28, Ch. 3] by using Diophantine approximation that every nonlattice string in $\mathbb{R}=\mathbb{Q}_{\infty}$ can be approximated by a sequence of lattice strings with oscillatory periods increasing to infinity. It follows that the complex dimensions of an archimedean nonlattice string are quasiperiodically distributed (in a very precise sense, that is explained in loc. cit.) because the complex dimensions of archimedean lattice strings are periodically distributed along finitely many vertical lines. Clearly, there is nothing of this kind in the nonarchimedean case since $p$-adic self-similar strings are necessarily lattice.

\section{Rationality of the Geometric Zeta Function}

In this section, we show that the geometric zeta function of a $p$-adic self-similar string is always rational (after an appropriate change of variable). It will follow (see Theorem 8.3) that not only the poles (i.e., the complex dimensions of $\mathcal{L}_{p}$ ) but also the zeros of $\zeta_{\mathcal{L}_{p}}$ are periodically distributed.

We introduce some necessary notation. First, by Lemma 6.2, we can write

$$
r_{j}=p^{-n_{j}}, \quad \text { with } \quad n_{j} \in \mathbb{N}^{*} \text { for } j=1,2, \ldots, N .
$$

Second, we write

$$
g_{k}=\mu_{H}\left(G_{k}\right)=p^{-m_{k}}, \quad \text { with } \quad m_{k} \in \mathbb{N}^{*} \quad \text { for } \quad k=1,2, \ldots, K .
$$

Third, let

$$
d=\operatorname{gcd}\left\{n_{1}, \ldots, n_{N}, m_{1}, \ldots, m_{K}\right\} .
$$

Then there exist positive integers $n_{j}^{\prime}$ and $m_{k}^{\prime}$ such that

$$
n_{j}=d n_{j}^{\prime} \quad \text { and } \quad m_{k}=d m_{k}^{\prime} \quad \text { for } \quad j=1, \ldots, N \quad \text { and } k=1, \ldots, K \text {. }
$$

\footnotetext{
${ }^{13}$ See, e.g., Examples 6.510 .7 and Theorem 8.1
} 
Finally, we set14

$$
p^{d}=1 / r .
$$

Without loss of generality, we may assume that the scaling ratios $r_{j}$ and the gaps $g_{k}$ are written in nonincreasing order as in Equations (6.1) and (6.4), respectively; so that

$$
0<n_{1}^{\prime} \leq n_{2}^{\prime} \leq \cdots \leq n_{N}^{\prime} \quad \text { and } \quad 0<m_{1}^{\prime} \leq m_{2}^{\prime} \leq \cdots \leq m_{K}^{\prime}
$$

THEOREM 8.1. Let $\mathcal{L}_{p}$ be a p-adic self-similar string and $z=r^{s}$, with $r=p^{-d}$ as in Equation (8.2). Then the geometric zeta function $\zeta_{\mathcal{L}_{p}}$ of $\mathcal{L}_{p}$ is a rational function in $z$. Specifically,

$$
\zeta_{\mathcal{L}_{p}}(s)=\frac{\sum_{k=1}^{K} z^{m_{k}^{\prime}}}{1-\sum_{j=1}^{N} z^{n_{j}^{\prime}}}
$$

where $m_{k}^{\prime}, n_{j}^{\prime} \in \mathbb{N}^{*}$ are given by Equation (8.1).

Definition 8.2. Let $\mathbf{p}=\frac{2 \pi}{d \log p}$. Then $\mathbf{p}$ is called the oscillatory period of $\mathcal{L}_{p}$.

8.1. Periodicity of the Poles and the Zeros of $\zeta_{\mathcal{L}_{p}}$. The following result (also from [18) is the nonarchimedean counterpart of [28, Thms. 2.17 and 3.6], which provide the rather subtle structure of the complex dimensions of archimedean self-similar strings. It is significantly simpler, however, due to the fact that nonlattice $p$-adic self-similar strings do not exist.

To avoid any confusion, we stress that in the statement of the next theorem, $\zeta_{\mathcal{L}_{p}}$ is viewed as a function of the original complex variable $s$. Moreover, as was recalled in Remark 3.8, it follows from a theorem in 20. that the dimension of $\mathcal{L}_{p}$ defined as the Minkowski dimension $D=D_{\mathcal{L}_{p}}$ coincides with the abscissa of convergence of the Dirichlet series originally defining $\zeta_{\mathcal{L}_{p}}$ and denoted (as in Equation (3.4)) by $\sigma=\sigma_{\mathcal{L}_{p}}$. Furthermore, let $\delta$ be the similarity dimension of $\mathcal{L}_{p}$, i.e., the unique real (and hence, positive) solution of the Moran equation $\sum_{j=1}^{N} r_{j}^{s}=1$; then $\delta=D$ by part (iii) of Theorem 8.3 below. Therefore, in the present case of $p$-adic self-similar strings, there is no need to distinguish between these various notions of 'fractal dimensions'.

TheOREM 8.3 (Structure of the complex dimensions). Let $\mathcal{L}_{p}$ be a nontrivial p-adic self-similar string. Then

(i) The complex dimensions of $\mathcal{L}_{p}$ and the zeros of $\zeta_{\mathcal{L}_{p}}$ are periodically distributed along finitely many vertical lines, with period $\mathbf{p}$, the oscillatory period of $\mathcal{L}_{p}$ (as given in Definition 8.2).

(ii) Furthermore, along a given vertical line, each pole (respectively, each zero) of $\zeta_{\mathcal{L}_{p}}$ has the same multiplicity.

(iii) Finally, the dimension $D$ of $\mathcal{L}_{p}$ is the only complex dimension that is located on the real axis. Moreover, $D$ is a simple pole of $\zeta_{\mathcal{L}_{p}}$ and is located on the right most vertical line. That is, $D$ is equal to the maximum of the real parts of the complex dimensions.

\footnotetext{
${ }^{14}$ Note that by construction, $r_{j}=r^{n_{j}^{\prime}}$ and $g_{k}=r^{m_{k}^{\prime}}$ for $j=1, \ldots, N$ and $k=1, \ldots, K$. Hence, $r=p^{-d}$ is the multiplicative generator in $(0,1)$ of the rank one group generated by $\left\{r_{1}, \ldots, r_{N}, g_{1}, \ldots, g_{K}\right\}$ (or, equivalently, by either $\left\{r_{1}, \ldots, r_{N}\right\}$ or $\left\{g_{1}, \ldots, g_{K}\right\}$ ).
} 
REMARK 8.4. The situation described above - specifically, the rationality of the zeta function in the variable $z=r^{s}$, with $r=p^{-d}$, and the ensuing periodicity of the poles and the zeros - is analogous to the one encountered for a curve (or more generally, a variety) over a finite field $\mathbb{F}_{p^{d}}$; see, e.g., Chapter 3 of [33. In this analogy, the prime number $p$ is the characteristic of the finite field, and the cardinality of the field, $p^{d}$, corresponds to $r^{-1}$, the reciprocal of the multiplicative generator of $\mathcal{L}_{p}$.

We next supplement the above results by stating a theorem (from [18, 19] and based on corresponding results in $[\mathbf{2 8}$, Chs. $2 \& 6]$ ) which will be very useful to us in $\$ 9$ in order to simplify the tube formula associated with a $p$-adic self-similar string 15

According to part (i) of Theorem 8.3 , there exist finitely many poles

$$
\omega_{1}, \ldots, \omega_{q},
$$

of $\zeta_{\mathcal{L}_{p}}$ with $\omega_{1}=D$ and $\Re\left(\omega_{q}\right) \leq \cdots \leq \Re\left(\omega_{2}\right)<D$, such that

$$
\mathcal{D}_{\mathcal{L}_{p}}=\left\{\omega_{u}+i n \mathbf{p}: n \in \mathbb{Z}, u=1, \ldots, q\right\} .
$$

Furthermore, each complex dimension $\omega+i n \mathbf{p}$ is simple (by parts (ii) and (iii) of Theorem 8.3) and the residue of $\zeta_{\mathcal{L}_{p}}(s)$ at $s=\omega+i n \mathbf{p}$ is independent of $n \in \mathbb{Z}$ and, in light of Equation (8.4), equal to

$$
\operatorname{res}\left(\zeta_{\mathcal{L}_{p}} ; \omega+i n \mathbf{p}\right)=\lim _{s \rightarrow \omega}(s-\omega) \frac{\sum_{k=1}^{K} r^{m_{k}^{\prime} s}}{1-\sum_{j=1}^{N} r^{n_{j}^{\prime} s}}=\frac{\sum_{k=1}^{K} r^{m_{k}^{\prime} \omega}}{\log r^{-1} \sum_{j=1}^{N} n_{j}^{\prime} r^{n_{j}^{\prime} \omega}} .
$$

In particular, this is the case for $\omega=D$. See [28, Ch. 6] for the general case.

THEOREM 8.5. (i) For each $u=1, \ldots, q$, the principal part of the Laurent series of $\zeta_{\mathcal{L}_{p}}(s)$ at $s=\omega_{u}+$ in $\mathbf{p}$ does not depend on $n \in \mathbb{Z}$.

(ii) Moreover, let $u \in\{1, \ldots, q\}$ be such that $\omega_{u}$ (and hence also $\omega_{u}+$ in $\mathbf{p}$, for every $n \in \mathbb{Z}$, by part (ii) of Theorem 8.3) is simple. Then the residue of $\zeta_{\mathcal{L}_{p}}(s)$ at $s=\omega_{u}+i n \mathbf{p}$ is independent of $n \in \mathbb{Z}$ and

$$
\operatorname{res}\left(\zeta_{\mathcal{L}_{p}} ; \omega_{u}+i n \mathbf{p}\right)=\frac{\sum_{k=1}^{K} r^{m_{k}^{\prime} \omega_{u}}}{\log r^{-1} \sum_{j=1}^{N} n_{j}^{\prime} n_{j}^{\prime \omega_{u}}} .
$$

In particular, this is the case for $\omega_{1}=D$.

Note that by contrast, in the lattice case of the archimedean theory of selfsimilar strings developed in [28, Chs. 2 and 3], one has to assume that the gap sizes (and not just the scaling ratios) are integral powers of $r$ in order to obtain the counterpart of Theorem 8.5 .

Remark 8.6 (Comparison with the archimedean case). Part (i) of Theorem 8.3. along with Theorem 8.1, shows that the theory of $p$-adic self-similar strings is simpler than its archimedean counterpart. Indeed, not only is it the case that every $p$-adic self-similar string $\mathcal{L}_{p}$ is lattice, but both the zeros and poles of $\zeta_{\mathcal{L}_{p}}(s)$ are periodically distributed along vertical lines, with the same period (because $\mathcal{L}_{p}$ is strongly lattice; see Theorem 17.4). By contrast, even if an archimedean selfsimilar string $\mathcal{L}$ is assumed to be 'lattice', then the zeros of $\zeta_{\mathcal{L}}(s)$ are usually not periodically distributed because the multiplicative group generated by the distinct

\footnotetext{
${ }^{15}$ In light of Theorems 8.1 and 8.3 Theorem 8.5 follows from a corresponding result in 28 .
} 
gap sizes need not be of rank one or coincide with the group generated by the distinct scaling ratios; see [28, Chs. 2 and 3]. In fact, from this point of view, only strongly lattice archimedean (or real) strings behave like $p$-adic self-similar strings.

\section{Exact Tube Formulas for $p$-Adic Self-Similar Strings}

In view of Equation (7.1), every $p$-adic self-similar string $\mathcal{L}_{p}$ is strongly languid, with $\kappa=0$ and $A=r_{N} g_{K}^{-1}$, in the notation of [28, Definition 5.3]. Indeed, Equation (7.1) implies that $\left|\zeta_{\mathcal{L}_{p}}(s)\right| \ll\left(r_{N}^{-1} g_{K}\right)^{-|\Re(s)|}$, as $\Re(s) \rightarrow-\infty$. Hence, we can apply the distributional tube formula without error term (i.e., the last part of Theorem 5.1 and of Corollary 5.2) with $W=\mathbb{C}$. Since by Theorem 7.4, $\mathcal{L}_{p}$ is a lattice string, we obtain (in light of Theorems 8.1, 8.3 and 8.5) the following simpler analogue of Theorem 8.25 in $\mathbf{2 8}: 16$

THEOREM 9.1 (Exact tube formulas for $p$-adic self-similar fractal strings). Let $\mathcal{L}_{p}$ be a p-adic self-similar string with simple complex dimensions. Then, for all $\varepsilon$ with $0<\varepsilon<g_{K} r_{N}^{-1}$, the volume $V_{\mathcal{L}_{p}}(\varepsilon)$ is given by

$$
V_{\mathcal{L}_{p}}(\varepsilon)=\sum_{\omega \in \mathcal{D}} c_{\omega} \varepsilon^{1-\omega}
$$

where $c_{\omega}=\frac{\operatorname{res}\left(\zeta_{\mathcal{L}_{p}} ; \omega\right)}{p(1-\omega)}$ for each $\omega \in \mathcal{D}=\mathcal{D}_{\mathcal{L}_{p}}(\mathbb{C})$.

COROLlaRY 9.2. Let $\mathcal{L}_{p}$ be a p-adic self-similar string with multiplicative generator $r$. Assume that all the complex dimensions of $\mathcal{L}_{p}$ are simple. Then, for all $\varepsilon$ with $0<\varepsilon<g_{K} r_{N}^{-1}$, the volume $V_{\mathcal{L}_{p}}(\varepsilon)$ is given by the following exact distributional tube formula:

$$
V_{\mathcal{L}_{p}}(\varepsilon)=\sum_{u=1}^{q} \varepsilon^{1-\omega_{u}} G_{u}\left(\log _{1 / r} \varepsilon^{-1}\right),
$$

where $1 / r=p^{d}$ (as in Equation (8.2)), and for each $u=1, \ldots, q, G_{u}$ is a real-valued periodic function of period 1 on $\mathbb{R}$ corresponding to the line of complex dimensions through $\omega_{u}\left(\omega_{1}=D>\Re\left(\omega_{2}\right) \geq \cdots \geq \Re\left(\omega_{q}\right)\right)$, and is given by the following (conditionally and also distributionally convergent) Fourier series:

$$
G_{u}(x)=\frac{\operatorname{res}\left(\zeta_{\mathcal{L}_{p}} ; \omega_{u}\right)}{p} \sum_{n \in \mathbb{Z}} \frac{e^{2 \pi i n x}}{1-\omega_{u}-i n \mathbf{p}}
$$

where (as in Equation (8.6) of Theorem 8.5),

$$
\operatorname{res}\left(\zeta_{\mathcal{L}_{p}} ; \omega_{u}\right)=\frac{\sum_{k=1}^{K} r^{m_{k}^{\prime} \omega_{u}}}{\log r^{-1} \sum_{j=1}^{N} n_{j}^{\prime} r^{n_{j}^{\prime} \omega_{u}}} .
$$

Moreover, $G_{u}$ is nonconstant and bounded.

\footnotetext{
${ }^{16}$ We note that instead, we could more generally apply parts (i) and (ii) of Theorem 5.1 in order to obtain a distributional tube formula with or without error term, valid without assuming that all of the complex dimensions of $\mathcal{L}_{p}$ are simple. This observation is used in the proof of Theorem 9.5
} 
Proof. That the explicit formula for $V_{\mathcal{L}_{p}}(\varepsilon)$ can be written as a sum over $\varepsilon^{1-\omega_{u}}$ times a periodic function of period 1 in $\log _{1 / r} \varepsilon^{-1}$ in case all complex dimensions are simple follows from Theorem 9.1, as does the formula for $G_{u}$. This latter function is clearly nonconstant. That it is bounded follows from [28, Formula (1.13)].

REMARK 9.3. In comparing our results with the corresponding results in Chapter 2 and $\S 8.4$ of [28], obtained for real self-similar fractal strings, the reader should keep in mind the following two facts: (i) the simplification brought upon by the "strong lattice property" of $p$-adic self-similar strings; see Theorem 8.5 and Remark 8.6 above. (ii) By construction, any $p$-adic self-similar string $\mathcal{L}_{p}$ (as defined in this paper) has total length $L$ equal to one: $L=\mu_{H}\left(\mathcal{L}_{p}\right)=\zeta_{\mathcal{L}_{p}}(1)=\mu_{H}\left(\mathbb{Z}_{p}\right)=1$. Indeed, for notational simplicity, we have assumed that the similarity transformations $\Phi_{j}(j=1, \ldots, N)$ are self-maps of the 'unit interval' $\mathbb{Z}_{p}$, rather than of an arbitrary 'interval' of length $L$ in $\mathbb{Q}_{p}$. Clearly, only minor adjustments are needed in order to deal with the case of an arbitrary interval.

REMARK 9.4. It would be interesting to obtain a geometric interpretation of the coefficients of the fractal tube formulas (9.1) and (9.2), in terms of nonarchimedean fractal curvatures, along the lines suggested by the work of [28] and [22, 23, 24] (in the archimedean setting). It would also be interesting to extend these results to higher-dimensional $p$-adic self-similar sets or tilings (as was done in the Euclidean case in loc. cit.).

THEOREM 9.5 (Truncated tube formula). Let $\mathcal{L}_{p}$ be an arbitrary $p$-adic selfsimilar string with multiplicative generator $r$. Then, for all $\varepsilon$ with $0<\varepsilon<g_{K} r_{N}^{-1}$,

$$
V_{\mathcal{L}_{p}}(\varepsilon)=\varepsilon^{1-D}\left(G\left(\log _{1 / r} \varepsilon^{-1}\right)+o(1)\right),
$$

where $o(1) \rightarrow 0$ as $\varepsilon \rightarrow 0^{+}$and $G=G_{1}$ is the nonconstant, bounded periodic function of period 1 given by Equation (9.3) of Theorem 9.2 (with $u=1$ and $\left.\omega_{1}=D\right)$.

PRoOF. This follows from the method of proof of Corollary 8.27 in [28 in the easy case of a lattice string and with $2 \varepsilon$ replaced by $\varepsilon$ and with $L:=1$; see Remark 9.3. In particular, we have the following 'truncated tube formula':

$$
V_{\mathcal{L}_{p}}(\varepsilon)=\varepsilon^{1-D} G\left(\log _{1 / r} \varepsilon^{-1}\right)+E(\varepsilon),
$$

where $E(\varepsilon)$ is an error term that can be estimated much as in loc. cit. In particular, there exists $\delta>0$ such that $\varepsilon^{-(1-D)} E(\varepsilon)=O\left(\varepsilon^{\delta}\right)$, as $\varepsilon \rightarrow 0^{+}$.

Furthermore, since we limit ourselves here to the first line of complex dimensions, and since those complex dimensions are always simple (by parts (ii) and (iii) of Theorem 8.3), we do not have to assume (as in Theorem 9.1 and Corollary 9.2) that all the complex dimensions of $\mathcal{L}_{p}$ are simple in order for Equation (9.5) and the corresponding error estimate for $E(\varepsilon)$ to be valid.

More specifically, we note that Equation (9.5) and the corresponding error estimate for $E(\varepsilon)$ (namely, $\delta>0$ and so $E(\varepsilon)=o\left(\varepsilon^{-(1-D)}\right)$ as $\varepsilon \rightarrow 0^{+}$) follow from the first part of Theorem 5.1 (the explicit tube formula with error term, applied to a suitable window), along with the fact that the complex dimensions on the rightmost vertical line $\Re(s)=D$ are simple (according to parts (ii) and (iii) of Theorem 8.3). Here, since $\mathcal{L}_{p}$ is a lattice string, we can simply choose the screen $S$ to be a vertical line lying strictly between $\Re(s)=D$ and the next vertical line of complex dimensions (if such a line exists). 


\section{The Average Minkowski Content}

The (inner) Minkowski dimension and the (inner) Minkowski content of a $p$ adic fractal string $\mathcal{L}_{p}$ (or, equivalently, of its metric boundary $\beta \mathcal{L}_{p}$, see Definition 4.1) are defined exactly as the corresponding notion for a real fractal string (see [28, Definition 1.2), except for the fact that we use the definition of $V(\varepsilon)=V_{\mathcal{L}_{p}}(\varepsilon)$ provided in Equation (4.3) of 4 More specifically, the Minkowski dimension of $\mathcal{L}_{p}$ is given by

$$
D_{M}:=\inf \left\{\alpha \geq 0: V_{\mathcal{L}_{p}}(\varepsilon)=O\left(\varepsilon^{1-\alpha}\right) \text { as } \varepsilon \rightarrow 0^{+}\right\} .
$$

Furthermore, $\mathcal{L}_{p}$ is said to be Minkowski measurable, with Minkowski content $\mathcal{M}$, if the limit

$$
\mathcal{M}=\lim _{\varepsilon \rightarrow 0^{+}} V_{\mathcal{L}_{p}}(\varepsilon) \varepsilon^{-\left(1-D_{M}\right)}
$$

exists in $(0, \infty)$. Otherwise, $\mathcal{L}_{p}$ is said to be Minkowski nonmeasurable.

REMARK 10.1. Note that since $V_{\mathcal{L}_{p}}(\varepsilon)=\mathcal{V}_{\mathcal{L}_{p}}(\varepsilon)-\mu_{H}\left(\beta \mathcal{L}_{p}\right)$ in light of Equation (4.3), there is an analogy between the above definition of the Minkowski dimension and that of "exterior dimension", which is used in chaos theory to study certain archimedean 'fat fractals' (dynamically defined fractals with positive Lebesgue measure); see, e.g., $\mathbf{7}$ and the survey article [32. In the present nonarchimedean case, however, for any $p$-adic fractal string, it is necessary to substract $\mu_{H}\left(\beta \mathcal{L}_{p}\right)$ from $\mathcal{V}_{\mathcal{L}_{p}}(\varepsilon)$. Indeed, otherwise, the metric boundary of every $p$-adic string (even a single interval) would be a 'fat fractal'; see [20 and Remark 4.4 above.

The next result follows from the truncated tube formula provided in Theorem 9.5. along with the corresponding error estimate.

TheOREM 10.2. A p-adic self-similar string $\mathcal{L}_{p}$ is never Minkowski measurable. Moreover, it has multiplicatively periodic oscillations of order $D$ in its geometry.

Proof. This follows immediately from Theorem 9.5 and the fact that $G=G_{1}$ is a nonconstant periodic function, which implies (in light of Equation (9.4)) that the limit of $\varepsilon^{-(1-D)} V_{\mathcal{L}_{p}}(\varepsilon)$ does not exist as $\varepsilon \rightarrow 0^{+}$.

According to Theorem 10.2, a $p$-adic self-similar string does not have a welldefined Minkowski content, because it is not Minkowski measurable. Nevertheless, as we shall see in Theorem 10.4 below, it does have a suitable 'average content' $\mathcal{M}_{a v}$, in the following sense:

Definition 10.3. Let $\mathcal{L}_{p}$ be a $p$-adic fractal string of dimension $D$. The average Minkowski content, $\mathcal{M}_{a v}$, is defined by the logarithmic Cesaro average

$$
\mathcal{M}_{a v}=\mathcal{M}_{a v}\left(\mathcal{L}_{p}\right):=\lim _{T \rightarrow \infty} \frac{1}{\log T} \int_{1 / T}^{1} \varepsilon^{-(1-D)} V_{\mathcal{L}_{p}}(\varepsilon) \frac{d \varepsilon}{\varepsilon},
$$

provided this limit exists and is a finite positive real number.

THEOREM 10.4. Let $\mathcal{L}_{p}$ be a p-adic self-similar string of dimension D. Then the average Minkowski content of $\mathcal{L}_{p}$ exists and is given by the finite positive number

$$
\mathcal{M}_{a v}=\frac{1}{p(1-D)} \operatorname{res}\left(\zeta_{\mathcal{L}_{p}} ; D\right)=\frac{1}{p(1-D)} \frac{\sum_{k=1}^{K} r^{m_{k}^{\prime} D}}{\log r^{-1} \sum_{j=1}^{N} n_{j}^{\prime} r^{n_{j}^{\prime} D}} .
$$


Proof. In light of (the proof of) Theorem 9.5, we have for all $0<\varepsilon \leq 1$ and for some $\delta>0$,

$$
\varepsilon^{-(1-D)} V(\varepsilon)=G\left(\log _{1 / r} \varepsilon^{-1}\right)+O\left(\varepsilon^{\delta}\right),
$$

where $G$ is the nonconstant and bounded periodic function of period 1 given by Equation (9.3) of Theorem 9.2 (with $u=1$ and $\omega_{1}=D$ ). (See Equation (9.5) and the text surrounding it.) Noting that

$$
\lim _{T \rightarrow \infty} \frac{1}{\log T} \int_{1 / T}^{1} \varepsilon^{\delta} \frac{d \varepsilon}{\varepsilon}=0,
$$

and that each oscillatory term of $G_{1}$ (for $n \neq 0$ in (9.3), $n \in \mathbb{Z}$ ) gives a vanishing contribution as well,

we conclude that

$$
\lim _{T \rightarrow \infty} \frac{1}{\log T} \int_{1 / T}^{1} \varepsilon^{i n / \log r} \frac{d \varepsilon}{\varepsilon}=0
$$

$$
\lim _{T \rightarrow \infty} \frac{1}{\log T} \int_{1 / T}^{1} \varepsilon^{-(1-D)} V_{\mathcal{L}_{p}}(\varepsilon) \frac{d \varepsilon}{\varepsilon}
$$

gives the constant coefficient of $G=G_{1}$.

REMARK 10.5. Definition 10.3 and Theorem 10.4 are the exact nonarchimedean counterpart of [28], Definition 8.29 and Theorem 8.30.

ExAmple 10.6 (Nonarchimedean Cantor string). The average Minkowski content of the nonarchimedean Cantor string $\mathcal{C S}_{3}$ is given by

$$
\mathcal{M}_{a v}\left(\mathcal{C S}_{3}\right)=\frac{1}{6(\log 3-\log 2)}
$$

Indeed, we have seen in Example 3.9 that $D=\log _{3} 2, \operatorname{res}\left(\zeta_{\mathcal{C S}_{3}} ; D\right)=1 / 2 \log 3$ and $p=3$.

EXAMPLE 10.7 (Nonarchimedean Fibonacci string). Let $\Phi_{1}$ and $\Phi_{2}$ be the two affine similarity contraction mappings of $\mathbb{Z}_{2}$ given (much as in $₫ 6$, with $N=p=2$ ) by

$$
\Phi_{1}(x)=2 x \quad \text { and } \quad \Phi_{2}(x)=1+4 x,
$$

with the respective scaling ratios $r_{1}=1 / 2$ and $r_{2}=1 / 4$. The associated 2-adic selfsimilar string (introduced in [18]) with generator $G=3+4 \mathbb{Z}_{2}$ is called the nonarchimedean Fibonacci string and denoted by $\mathcal{F S}_{2}$ (compare with the archimedean counterpart discussed in [28, $\S 2.3 .2]$ ). It is given by the sequence $\mathcal{F} \mathcal{S}_{2}=l_{1}, l_{2}, l_{3}, \ldots$ and consists (for $m=1,2, \ldots$ ) of intervals of lengths $l_{m}=2^{-(m+1)}$ with multiplicities $f_{m}$, the Fibonacci numbers. (Recall that these numbers are defined by the recursive formula: $f_{m+1}=f_{m}+f_{m-1}, f_{0}=0$ and $f_{1}=1$.) Alternatively, in the spirit of Theorem 6.4 the nonarchimedean Fibonacci string is the bounded open subset of $\mathbb{Z}_{2}$ given by the following disjoint union of 2-adic intervals (necessarily its 2-adic convex components):

$$
\mathcal{F S}_{2}=\left(3+4 \mathbb{Z}_{2}\right) \cup\left(6+8 \mathbb{Z}_{2}\right) \cup\left(12+16 \mathbb{Z}_{2}\right) \cup\left(13+16 \mathbb{Z}_{2}\right) \cup \cdots .
$$

By Theorem [7.1, the geometric zeta function of $\mathcal{F} \mathcal{S}_{2}$ is given (almost exactly as for the archimedean Fibonacci string, cf. loc. cit.) by

\footnotetext{
${ }^{17}$ The minor difference between the two geometric zeta functions is due to the fact that the real Fibonacci string $\mathcal{F S}$ in [28, $§ 2.3 .2$ and Exple. 8.32] has total length 4 whereas the present 2-adic Fibonacci string $\mathcal{F S}_{2}$ has total length 1; see also part (ii) of Remark 9.3 above.
} 


$$
\zeta_{\mathcal{F} \mathcal{S}_{2}}(s)=\frac{4^{-s}}{1-2^{-s}-4^{-s}} .
$$

Hence, the set of complex dimensions of $\mathcal{F} \mathcal{S}_{2}$ is given by

$$
\mathcal{D}_{\mathcal{F} \mathcal{S}_{2}}=\{D+i n \mathbf{p}: n \in \mathbb{Z}\} \cup\{-D+i(n+1 / 2) \mathbf{p}: n \in \mathbb{Z}\}
$$

with $D=\log _{2} \phi$, where $\phi=(1+\sqrt{5}) / 2$ is the golden ratio, and $\mathbf{p}=2 \pi / \log 2$, the oscillatory period of $\mathcal{F} \mathcal{S}_{2}$; see Figure 4. Moreover, a simple computation shows that

$$
\operatorname{res}\left(\zeta_{\mathcal{F} \mathcal{S}_{2}} ; D+i n \mathbf{p}\right)=\frac{3-\phi}{5 \log 2}
$$

and

$$
\operatorname{res}\left(\zeta_{\mathcal{F} \mathcal{S}_{2}} ;-D+i(n+1 / 2) \mathbf{p}\right)=\frac{2+\phi}{5 \log 2}
$$

independently of $n \in \mathbb{Z}$.

We refer the interested reader to $\mathbf{1 8}$ for additional information concerning the nonarchimedean Fibonacci string.

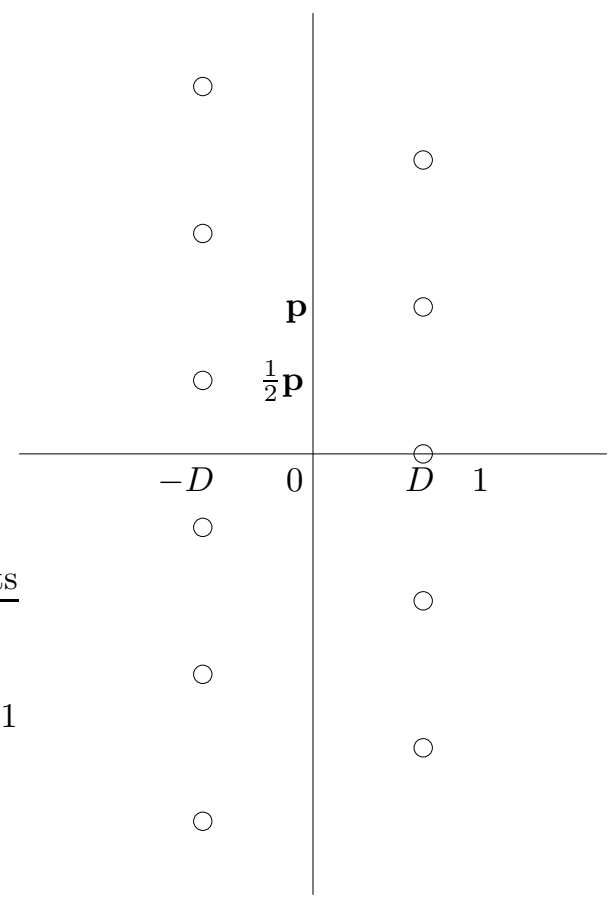

Figure 4. The complex dimensions of the 2-adic Fibonacci string $\mathcal{F S}_{2}$. Here, $D=\log _{2} \phi$ and $\mathbf{p}=2 \pi / \log 2$.

Note that $\zeta_{\mathcal{F} \mathcal{S}_{2}}$ does not have any zero (in the variable $s$ ) since the equation $4^{-s}=0$ does not have any complex solution. Moreover, in agreement with Theorem 8.1. $\zeta_{\mathcal{F} \mathcal{S}_{2}}$ is a rational function of $z=2^{-s}$, i.e.,

$$
\zeta_{\mathcal{F} \mathcal{S}_{2}}(s)=\frac{z^{2}}{1-z-z^{2}} .
$$


Since, in light of (10.8), the complex dimensions of $\mathcal{F} \mathcal{S}_{2}$ are simple, we may apply either Corollary 5.2 or Corollary 9.2 in order to obtain the following exact fractal tube formula for the nonarchimedean Fibonacci string 18

$$
\begin{aligned}
V_{\mathcal{F S}_{2}}(\varepsilon) & =\frac{1}{2} \sum_{\omega \in \mathcal{D}_{\mathcal{F} \mathcal{S}_{2}}} \operatorname{res}\left(\zeta_{\mathcal{F} \mathcal{S}_{2}} ; \omega\right) \frac{\varepsilon^{1-\omega}}{1-\omega} \\
& =\varepsilon^{1-D} G_{1}\left(\log _{2} \varepsilon^{-1}\right)+\varepsilon^{1+D-i \mathbf{p} / 2} G_{2}\left(\log _{2} \varepsilon^{-1}\right),
\end{aligned}
$$

where, in light Equation (10.5) and of the values of $\operatorname{res}\left(\zeta_{\mathcal{F} \mathcal{S}_{2}} ; \omega\right)$ provided in Equations (10.6) and (10.7), $G_{1}$ and $G_{2}$ are bounded periodic functions of period 1 on $\mathbb{R}$ given by their respective (conditionally convergent) Fourier series

$$
G_{1}(x)=\frac{3-\phi}{10 \log 2} \sum_{n \in \mathbb{Z}} \frac{e^{2 \pi i n x}}{1-D-i n \mathbf{p}}
$$

and

$$
G_{2}(x)=\frac{2+\phi}{10 \log 2} \sum_{n \in \mathbb{Z}} \frac{e^{2 \pi i n x}}{1+D-i(n+1 / 2) \mathbf{p}} .
$$

Note that the above Fourier series for $G_{1}$ and $G_{2}$ are conditionally (and also distributionally) convergent, for all $x \in \mathbb{R}$. Furthermore, the explicit fractal tube formula (10.9) for $\mathcal{F S}_{2}$ actually holds pointwise and not just distributionally, as the interested reader may verify via a direct computation. The average Minkowski content of $\mathcal{F S}_{2}$ is given by

$$
\mathcal{M}_{a v}=\mathcal{M}_{a v}\left(\mathcal{F S}_{2}\right)=\frac{1}{2(\phi+2)(\log 2-\log \phi)},
$$

where $\phi=\frac{1+\sqrt{5}}{2}$ is the golden ratio. Indeed, since $D=\log _{2} \phi$, we deduce from Equation (10.6) with $n=0$ that

$$
\operatorname{res}\left(\zeta_{\mathcal{F} \mathcal{S}_{2}} ; D\right)=\frac{1}{(\phi+2) \log 2} .
$$

Hence, the above expression for $\mathcal{M}_{a v}$ follows from Theorem 10.4 with $p=2$. Furthermore, note that $\log 2-\log \phi=\log (\sqrt{5}-1)$. Therefore, $\mathcal{M}_{a v}$ can be rewritten as follows:

$$
\mathcal{M}_{a v}=\frac{1}{(5+\sqrt{5}) \log (\sqrt{5}-1)}
$$

\section{References}

1. A. S. Besicovitch and S. J. Taylor, On the complementary intervals of a linear closed set of zero Lebesgue measure, J. London Math. Soc. 29 (1954), 449-459.

2. B. Dragovich, Adelic harmonic oscillator, Internat. J. Mod. Phys. A 10 (1995), 2349-2365.

3. B. Dragovich, A. Yu. Khrennikov, S. V. Kozyrev and I. V. Volovich, On $p$-adic mathematical physics, p-Adic Numbers, Ultrametric Analysis and Applications No. 1, 1 (2009), 1-17.

4. C. J. Everett and S. Ulam, On some possibilities of generalizing the Lorentz group in the special relativity theory, J. Combinatorial Theory 1 (1966), 248-270.

5. K. J. Falconer, Fractal Geometry: Mathematical foundations and applications, Wiley, Chichester, 1990.

6. G. W. Gibbons and S. W. Hawking (eds.), Euclidean Quantum Gravity, World Scientific Publ., Singapore, 1993.

\footnotetext{
${ }^{18}$ In light of Theorem 4.3 one can also directly derive this formula for $V_{\mathcal{F} \mathcal{S}_{2}}(\varepsilon)$, much as was done for $V_{\mathcal{C} \mathcal{S}_{3}}(\varepsilon)$ in Example 4.5, although with some more strenuous work.
} 
7. C. Grebogi, S. McDonald, E. Ott and J. York, Exterior dimension of fat fractals, Physics Lett. A No. 1, 110 (1985), 1-4.

8. S. W. Hawking and W. Israel (eds.), General Relativity: An Einstein centenary survey, Cambridge Univ. Press, Cambridge, 1979.

9. J. E. Hutchinson, Fractals and self-similarity, Indiana Univ. Math. J. 30 (1981), 713-747.

10. N. Koblitz, p-adic Numbers, $p$-adic Analysis, and Zeta-functions, Springer-Verlag, New York, 1984.

11. A. Khrennikov, p-Adic Valued Distributions in Mathematical Physics, Kluwer Academic Publ., Dordrecht, 1994.

12. M. L. Lapidus, Fractal drum, inverse spectral problems for elliptic operators and a partial resolution of the Weyl-Berry conjecture, Trans. Amer. Math. Soc. 325 (1991), 465-529.

13. M. L. Lapidus, Spectral and fractal geometry: From the Weyl-Berry conjecture for the vibrations of fractal drums to the Riemann zeta-function, in: Differential Equations and Mathematical Physics (Birmingham, 1990), C. Bennewitz (ed.), Academic Press, New York, 1992, pp. 151-182.

14. M. L. Lapidus, Vibrations of fractal drums, the Riemann hypothesis, waves in fractal media, and the Weyl-Berry conjecture, in: Ordinary and Partial Differential Equations (B. D. Sleeman and R. J. Jarvis, eds.), Vol. IV, Proc. Twelfth Internat. Conf. (Dundee, Scotland, UK, June 1992), Pitman Research Notes in Math. Series, Vol. 289, Longman Scientific and Technical, London, 1993, pp. 126-209.

15. M. L. Lapidus, In Search of the Riemann Zeros: Strings, fractal membranes and noncommutative spacetimes, Amer. Math. Soc., Providence, RI, 2008.

16. M. L. Lapidus, J. Lévy Véhel and J. A. Rock, Fractal strings and multifractal zeta functions, Lett. Math. Phys. No. 1, 88 (2009), 101-129 (special issue dedicated to the memory of Moshe Flato). (Springer Open Access: DOI 10.1007/s11050090302y.)

17. M. L. Lapidus and Lũ Hùng, Nonarchimedean Cantor set and string, J. Fixed Point Theory and Appl. 3 (2008), 181-190. (Special issue dedicated to the Jubilee of V. I. Arnol'd, Vol. I.)

18. M. L. Lapidus and Lũ Hùng, Self-similar $p$-adic fractal strings and their complex dimensions, p-Adic Numbers, Ultrametric Analysis and Applications No. 2, 1 (2009), 167-180.

19. M. L. Lapidus and Lũ Hùng, The geometry of $p$-adic fractal strings: A comparative survey, in: Advances in Non-Archimedean Analysis, Contemporary Math., Vol. 551, Amer. Math. Soc., Providence, RI, 2010, pp. 163-206.

20. M. L. Lapidus, Lũ Hùng and M. van Frankenhuijsen, Minkowski dimension and explicit tube formulas for $p$-adic fractal strings, preprint, 2012.

21. M. L. Lapidus and H. Maier, The Riemann hypothesis and inverse spectral problems for fractal strings, J. London Math. Soc. (2) 52 (1995), 15-34.

22. M. L. Lapidus and E. P. J. Pearse, Tube formulas and complex dimensions of self-similar tilings, Acta Mathematicae Applicandae, 112 (2010), 91-137.

23. M. L. Lapidus and E. P. J. Pearse, Tube formulas for self-similar fractals, in: Analysis on Graphs and Its Applications (P. Exner et al., eds.), Proc. Sympos. Pure Math., Vol. 77, Amer. Math. Soc., Providence, RI, 2008, pp. 211-230.

24. M. L. Lapidus, E. P. J. Pearse and S. Winter, Pointwise tube formulas for fractal sprays and self-similar strings with arbitrary generators, Advances in Math. 227 (2011), 1349-1398. (E-print, arXiv: 1006.3807v2 [math. MG], 2011.)

25. M. L. Lapidus and C. Pomerance, The Riemann zeta-function and the one-dimensional WeylBerry conjecture for fractal drums, Proc. London Math. Soc. (3) 66 (1993), 41-69.

26. M. L. Lapidus and C. Pomerance, Counterexamples to the modified Weyl-Berry conjecture on fractal drums, Math. Proc. Cambridge Philos. Soc. 119 (1996), 167-178.

27. M. L. Lapidus and M. van Frankenhuijsen, Fractal Geometry and Number Theory: Complex dimensions of fractal strings and zeros of zeta functions, Birkhhäuser, Boston, 2000.

28. M. L. Lapidus and M. van Frankenhuijsen, Fractal Geometry, Complex Dimensions and Zeta Functions: Geometry and spectra of fractal strings, Springer Monographs in Mathematics, Springer-Verlag, New York, 2006. (Second revised and enlarged edition, 2012.)

29. P. A. P. Moran, Additive functions of intervals and Hausdorff measure, Math. Proc. Cambridge Philos. Soc. 42 (1946), 15-23.

30. J. Neukirch, Algebraic Number Theory, A Series of Comprehensive Studies in Mathematics, Springer-Verlag, New York, 1999. 
31. L. Notale, Fractal Spacetime and Microphysics: Towards a theory of scale relativity, World Scientific Publ., Singapore, 1993.

32. E. Ott, Fat Fractals, in: Chaos in Dynamical Systems, Cambridge Univ. Press, New York, 1993, pp. 97-100 (§3.9).

33. A. N. Parshin and I. R. Shafarevich (eds.), Number Theory, Vol. I, Introduction to Number Theory, Encyclopedia of Mathematical Sciences, Vol. 49, Springer-Verlag, Berlin, 1995. (Written by Yu. I. Manin and A. A. Panchishkin.)

34. E. P. J. Pearse, Canonical self-similar tilings by iterated function systems, Indiana Univ. Math. J. 56 (2007), 3151-3169.

35. E. P. J. Pearse and S. Winter, Geometry of canonical self-similar tilings, Rocky Mountain J. Math., in press, 2012. (E-print, arXiv: 0811.2187, 2009.)

36. R. Rammal, G. Toulouse and M. A. Virasoro, Ultrametricity for physicists, Rev. Mod. Phys. 58 (1986), 765-788.

37. A. M. Robert, A Course in p-adic Analysis, Graduate Texts in Mathematics, Springer-Verlag, New York, 2000.

38. W. H. Schikhof, Ultrametric Calculus: An introduction to p-adic analysis, Cambridge Studies in Advanced Mathematics, Cambridge Univ. Press, Cambridge, 1984.

39. J.-P. Serre, A Course in Arithmetic, English translation, Springer-Verlag, Berlin, 1973.

40. V. S. Vladimirov, I. V. Volovich and E. I. Zelenov, p-Adic Analysis and Mathematical Physics, World Scientific Publ., Singapore, 1994.

41. I. V. Volovich, Number theory as the ultimate physical theory, preprint, CERN-TH. 4791 , 1987.

42. J. A. Wheeler and K. W. Ford, Geons, Black Holes, and Quantum Foam: A life in Physics, W. W. Norton, New York, 1998.

Department of Mathematics, University of California, Riverside, CA 92521-0135

E-mail address: lapidus@math.ucr.edu

Department of Mathematics, Hawaís Pacific University, Honolulu, Hi 96813-2785

E-mail address: hlu@hpu.edu

Department of Mathematics, Utah Valley University, Orem, Ut 84058-5999

E-mail address: vanframa@uvu.edu 


$$
----\ldots \text { dots }
$$

$\begin{array}{llllllll}11 & 12 & 12 & 13 & 13 & 13 & 13 & 1 n\end{array}$ 
$\mathrm{C} 0$

$\mathrm{C} 1$ 
O

O

O

O

10

O

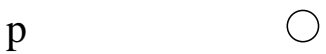

0

D

O

O

$\bigcirc$

O

0

0 


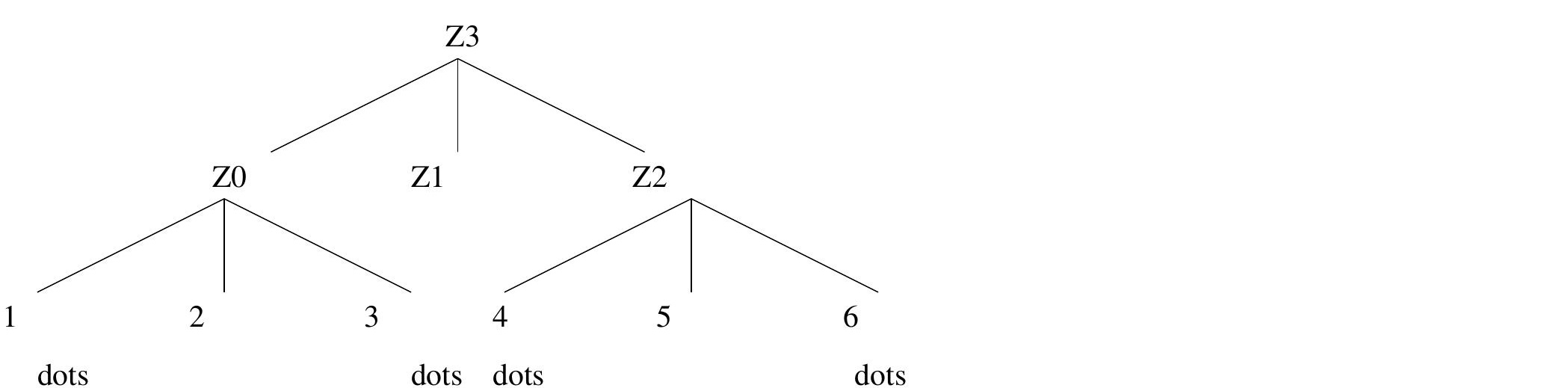




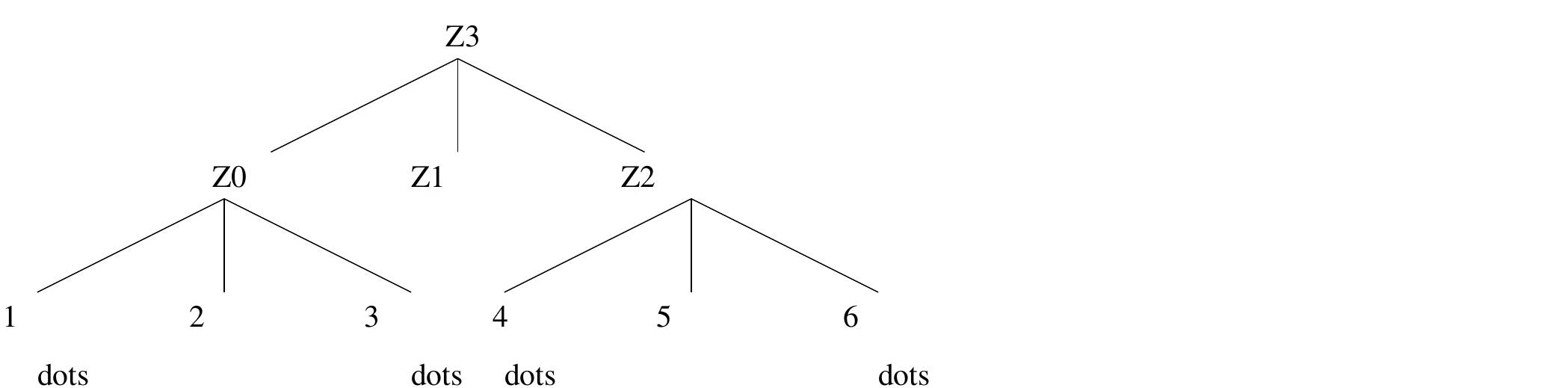



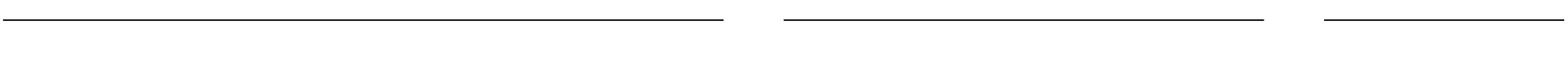

$--$

$\begin{array}{lllllll}11 & 12 & 13 & 14 & 15 & 16 & \ln \end{array}$

$|+|$ dots

